\title{
Charge and Spin Currents in Ferromagnet-Insulator-Superconductor Tunneling Junctions Using Hg-1223 High- $T_{c}$ Superconductor
}

\author{
Michihide Kitamura, Yoshitaka Uchiumi, and Akinobu Irie \\ Department of Electrical and Electronic System Engineering, Utsunomiya University, 7-1-2 Yoto, Utsunomiya, \\ Tochigi 321-8585, Japan \\ Correspondence should be addressed to Michihide Kitamura; kitamura@cc.utsunomiya-u.ac.jp
}

Received 25 April 2014; Revised 7 July 2014; Accepted 8 July 2014; Published 11 August 2014

Academic Editor: Zigang Deng

Copyright (C) 2014 Michihide Kitamura et al. This is an open access article distributed under the Creative Commons Attribution License, which permits unrestricted use, distribution, and reproduction in any medium, provided the original work is properly cited.

\begin{abstract}
Charge and spin currents along the $c$-axis in ferromagnet-insulator-superconductor (F/I/S) tunneling junctions have been studied within the framework of the tunneling Hamiltonian model. As a superconductor $S, \mathrm{HgBa}_{2} \mathrm{Ca}_{2} \mathrm{Cu}_{3} \mathrm{O}_{8+\delta}(\mathrm{Hg}-1223)$ with $\delta=0.4$ copper-oxide high- $T_{c}$ superconductor has been selected, and as a ferromagnet $F$, Fe metal with bcc structure has been selected for simplicity. The electronic structures of above materials have been calculated on the basis of the band theory using the spin-polarized self-consistent-field data for the atomic orbital energies and the universal tight-binding parameters (UTBP) for the interactions. For the $\eta_{\uparrow}$ and $\eta_{\downarrow}\left(=1-\eta_{\uparrow}\right)$ defined in the present paper, which are tunneling probabilities of the majority and the minority spin electrons, it is shown that the condition $\eta_{\uparrow}=\eta_{\downarrow}$ means the standard F/I/S tunneling junction with a nonmagnetic insulating layer, and the condition $\eta_{\uparrow} \neq \eta_{\downarrow}$ means the F/I/S tunneling junction with a magnetic insulating layer showing a detectable magnetization. We have found that the charge current and the differential conductance nearly remain the same as the change of $\eta_{\uparrow}$, but the spin current is largely changed due to the change of $\eta_{\uparrow}$. As an experimental method to detect the change of the spin current, the validity of an X-ray magnetic circular dichroism (XMCD) has been pointed out.
\end{abstract}

\section{Introduction}

In 1982, Blonder, Tinkham, and Klapwijk (BTK) presented a pioneering paper for an interface at the normal $(\mathrm{N})$ material and superconductor $(S)$, in which they proposed a simple theory for the current-voltage $(I-V)$ curves of normal (N)-superconducting (S) microconstriction contacts which describes the crossover from metallic to tunnel junction behavior [1]. Their model based on the Bogoliubov-de Gennes (BdG) equation, now called as "BTK model," worked well to understand the transmission and reflection of particle at N-S interface. Their results told us that the probability of Andreev reflection decreases with increasing the barrier strength $Z$ at the interface and the BTK model reaches to the tunneling Hamiltonian model due to the increasing $Z$ value. Based on the BTK model, Kashiwaya et al. theoretically studied the origin of zero-bias conductance peaks (ZBCPs) observed in the YBCO high- $T_{c}$ superconductors and found that the calculation is in good agreement with the experiment [2]. The ZBCPs are observed at the $I-V$ characteristics of the NS-interface. Within the framework of the BTK model, Kashiwaya et al. further studied the $I-V$ characteristics of $\mathrm{N} / \mathrm{I} / \mathrm{S}$ and $\mathrm{F} / \mathrm{I} / \mathrm{S}$ junctions $[3,4]$. Here it is noted that they have adopted the BTK model, so that the potential of the real insulating layer $I$ has been treated as a delta-functional and the tunneling electron has been regarded as a free particle under the effective mass approximation. Similar considerations have also been done by Annunziata et al. [5].

In the present paper, we consider the $c$-axis electron tunneling in the F/I/S tunneling junction using the $d$ wave high- $T_{c}$ superconductors. The insulating layer $I$ with a real dimension is considered here, so that the tunneling 
Hamiltonian model could be reliable for the present purpose because of a large $Z$-value. Furthermore, the electron is treated as not free but Bloch type, so that the electron states have been calculated on the basis of the band theory. It is well known that the superconducting energy gaps $\Delta$ of $d$ wave copper-oxide high- $T_{c}$ superconductors strongly depend on the wavenumber vector $\mathbf{k}$ characterizing the $\mathrm{CP}$, that is, $\Delta=\Delta_{\mathbf{k}}$, and that the $\mathbf{k}$-dependence of $\Delta_{\mathbf{k}}$ is given by $k_{x}^{2}-k_{y}^{2}$. It should be emphasized here that, in the tunneling Hamiltonian model adopted in the present paper, the $\mathbf{k}$ dependence of the $\Delta_{\mathbf{k}}$ is exactly taken into account in all the calculation procedures.

Recently, an interesting experimental paper has been presented by Takeshita et al. [6], in which they have measured the electrical resistivity of a $\mathrm{HgBa}_{2} \mathrm{Ca}_{2} \mathrm{Cu}_{3} \mathrm{O}_{8+\delta}$ (Hg-1223) polycrystalline sample under the high pressure and found the $T_{c}$ with $153.0 \mathrm{~K}$ at the hydrostatic pressure of $15 \mathrm{GPa}$. It was already reported and well known that the Hg-based copper-oxides high- $T_{c}$ superconductors $\mathrm{HgBa}_{2} \mathrm{Ca}_{n-1} \mathrm{Cu}_{n} \mathrm{O}_{2 n+2+\delta}$ with $n=1,2,3$ and 4, which are denoted as $\mathrm{Hg}-1201, \mathrm{Hg}-1212, \mathrm{Hg}-1223$, and $\mathrm{Hg}-1234$, show higher $T_{c}$ as compared with the other copper-oxides superconductors such as BSCCO and YBCO. The $T_{c}$ in $\mathrm{K}$ is $97,127,135$, and 127, respectively [7], for the $\mathrm{Hg}-1201$, $1212,-1223$, and -1234 , and the superconducting gap at low temperature region $\Delta(0)$ in $\mathrm{meV}$ is 33,50 , and 75 for the $\mathrm{Hg}$ $1201,-1212$, and -1223 , respectively [8]. It has been reported that the measured values of $\delta$ in high-T samples are approximately 0.06 in $\mathrm{Hg}-1201,0.22$ in $\mathrm{Hg}-1212$, and 0.4 in Hg1223 [9]. The $T_{c}$ of Hg-based copper-oxides superconductors is fairly higher than the liquid nitrogen temperature $T_{\mathrm{LN}}(=$ $77 \mathrm{~K}$ ), so it is surely expected that the superconductivity is well kept even in the case that the Hg-based copperoxides superconductors have been operated at $T_{\mathrm{LN}}$. From the experimental and the applicational points of view, in the present paper, the $\mathrm{Hg}-1223$ high- $T_{c}$ superconductor with $\delta=$ 0.4 , that is, $\mathrm{HgBa}_{2} \mathrm{Ca}_{2} \mathrm{Cu}_{3} \mathrm{O}_{8.4}$ whose $T_{c}$ is $135 \mathrm{~K}$, is selected as the superconducting $(\mathrm{S})$ layer. The sample temperature in the present calculations is fixed to $T_{\mathrm{LN}}$, so that the value of $T_{\mathrm{LN}} / T_{c}$ is 0.57 . The value of $\Delta(0)$ is $75 \mathrm{meV}$, so that the BCS curve for the energy gap $\Delta(\tau)$ as a function of the reduced temperature $\tau \equiv T / T_{c}$ gives a value of $70 \mathrm{meV}$ at $T=T_{\mathrm{LN}}$.

In the present paper, for simplicity, Fe metal with bcc structure has been adopted as a ferromagnetic $(\mathrm{F})$ layer. It is sure that the electron states in the vicinity of the Fermi level mainly come from $3 d$ orbital of the transition metals and that the density of states (DOS) that originated from the $3 d$ orbital shows a pointed structure meaning the localized nature, on the contrary to the DOS from $s$ and $p$ orbitals which show a broadened structure, that is, the extended nature. It may be sure that the effective mass approximation works well for the extended nature but not so for the localized one. This is just a reason why we have adopted the tunneling Hamiltonian model based on the electrons with the Bloch states.

\section{Theoretical}

The charge and spin currents, $I_{T}^{(+)}(V)$ and $I_{T}^{(-)}(V)$, as a function of an applied voltage $V$ can be evaluated by using
(A.8) and (A.9). In the following, therefore, we consider the tunneling current $i_{T, \sigma}(V)$ given by (A.9); that is,

$$
\begin{aligned}
& i_{T, \sigma}(V)=\frac{2 \pi e}{\hbar} \sum_{\mathbf{k}_{1}} \sum_{\mathbf{k}_{2}}\left|T_{\mathbf{k}_{1} \mathbf{k}_{2} \sigma}\right|^{2} g\left(\xi_{\mathbf{k}_{1} \sigma}^{(F)}, \xi_{\mathbf{k}_{2}}^{(S)}, \Delta_{\mathbf{k}_{2}}, e V\right), \\
& g\left(\xi_{\mathbf{k}_{1} \sigma}^{(F)}, \xi_{\mathbf{k}_{2}}^{(S)}, \Delta_{\mathbf{k}_{2}}, e V\right) \\
& =\left\{\left(f\left(\xi_{\mathbf{k}_{1} \sigma}^{(F)}\right)-f\left(E_{\mathbf{k}_{2}}\right)\right) \delta\left(\xi_{\mathbf{k}_{1} \sigma}^{(F)}+e V-E_{\mathbf{k}_{2}}\right)\right. \\
& \left.\quad+\left(f\left(\xi_{\mathbf{k}_{1} \sigma}^{(F)}\right)+f\left(E_{\mathbf{k}_{2}}\right)-1\right) \delta\left(\xi_{\mathbf{k}_{1} \sigma}^{(F)}+e V+E_{\mathbf{k}_{2}}\right)\right\} .
\end{aligned}
$$

Generally, $\left|T_{\mathbf{k}_{1} \mathbf{k}_{2} \sigma}\right|^{2}$ can be written as $\left|T_{\sigma}\right|^{2} \tau\left(\mathbf{k}_{1}, \mathbf{k}_{2}\right)$. Here $\left|T_{\sigma}\right|^{2}$ is a value depending only the spin state $\sigma$ and the $\tau\left(\mathbf{k}_{1}, \mathbf{k}_{2}\right)$ is a dimensionless value as a function of $\mathbf{k}_{1}$ and $\mathbf{k}_{2}$. As a value of $\tau\left(\mathbf{k}_{1}, \mathbf{k}_{2}\right)$, we consider here three cases; that is, the first one is the coherent tunneling case denoted by $\tau_{\text {Coh }}\left(\mathbf{k}_{1}, \mathbf{k}_{2}\right)$, the second one is the incoherent case $\tau_{\text {Inc }}\left(\mathbf{k}_{1}, \mathbf{k}_{2}\right)$, and the third one is the case based on the WKB approximation $\tau_{\mathrm{WKB}}\left(\mathbf{k}_{1}, \mathbf{k}_{2}\right)$. It is clear that $\tau_{\mathrm{Coh}}\left(\mathbf{k}_{1}, \mathbf{k}_{2}\right)=\delta_{\mathbf{k}_{1}, \mathbf{k}_{2}}$ and $\tau_{\text {Inc }}\left(\mathbf{k}_{1}, \mathbf{k}_{2}\right)=1$. In the WKB treatment, the momentum parallel to the junction plane is conserved in the tunneling processes [11]. If we use a geometrical configuration such that the axis normal to the junction plane is $z$-axis which is parallel to the $c$-axis, then $\tau_{\mathrm{WKB}}\left(\mathbf{k}_{1}, \mathbf{k}_{2}\right)$ can be written as $q_{1 z} q_{2 z} \delta_{k_{1 x}, k_{2 x}} \delta_{k_{1 y}, k_{2 y}}$, where $q_{1 z} \equiv k_{1 z} / k_{1 z}^{(\max )}$ and $q_{2 z} \equiv$ $k_{2 z} / k_{2 z}^{(\max )}$. Here it should be noted that two wavenumbers $k_{1 z}^{(\max )}$ and $k_{2 z}^{(\max )}$ have been introduced so as to satisfy the condition such that the $q_{1 z}$ and $q_{2 z}$ must be the dimensionless values. Namely, it must be emphasized that the amplitudes of $k_{1 z}^{(\max )}$ and $k_{2 z}^{(\max )}$ are not essential in the evaluation of $\tau_{\mathrm{WKB}}\left(\mathbf{k}_{1}, \mathbf{k}_{2}\right)$ and that the influence of the amplitudes $k_{1 z}^{(\max )}$ and $k_{2 z}^{(\max )}$ is included in the value of $\left|T_{\sigma}\right|^{2}$. From the above, the $i_{T, \sigma}(V)$ given by (1) can be rewritten as follows:

$$
i_{T, \sigma}(V)=\frac{2 \pi e}{\hbar} \sum_{\mathbf{k}_{2}}\left|T_{\sigma}\right|^{2} \Theta_{\sigma}^{(F)}\left(\xi_{\mathbf{k}_{2}}^{(S)}, \Delta_{\mathbf{k}_{2}}, e V\right) .
$$

For the function $\Theta_{\sigma}^{(F)}\left(\xi_{\mathbf{k}_{2}}^{(S)}, \Delta_{\mathbf{k}_{2}}, e V\right)$, there are three functions $\Theta_{\sigma}^{(F)}\left(\xi_{\mathbf{k}_{2}}^{(S)}, \Delta_{\mathbf{k}_{2}}, e V\right)_{\text {Coh }}, \quad \Theta_{\sigma}^{(F)}\left(\xi_{\mathbf{k}_{2}}^{(S)}, \Delta_{\mathbf{k}_{2}}, e V\right)_{\text {Inc }}$, and $\Theta_{\sigma}^{(F)}\left(\xi_{\mathbf{k}_{2}}^{(S)}, \Delta_{\mathbf{k}_{2}}, e V\right)_{\mathrm{WKB}}$ corresponding to the coherent, incoherent, and $\mathrm{WKB}$ cases. Those are given as follows:

$$
\begin{gathered}
\Theta_{\sigma}^{(F)}\left(\xi_{\mathbf{k}_{2}}^{(S)}, \Delta_{\mathbf{k}_{2}}, e V\right)_{\mathrm{Coh}}=g\left(\xi_{\mathbf{k}_{2} \sigma}^{(F)}, \xi_{\mathbf{k}_{2}}^{(S)}, \Delta_{\mathbf{k}_{2}}, e V\right) \\
\Theta_{\sigma}^{(F)}\left(\xi_{\mathbf{k}_{2}}^{(S)}, \Delta_{\mathbf{k}_{2}}, e V\right)_{\text {Inc }} \\
=\left\{\left\{f\left(E_{\mathbf{k}_{2}}-e V\right)-f\left(E_{\mathbf{k}_{2}}\right)\right\} D_{\sigma}^{(F)}\left(E_{\mathbf{k}_{2}}-e V\right)\right. \\
\left.\quad+\left\{f\left(E_{\mathbf{k}_{2}}\right)-f\left(E_{\mathbf{k}_{2}}+e V\right)\right\} D_{\sigma}^{(F)}\left(-E_{\mathbf{k}_{2}}-e V\right)\right\} \\
\Theta_{\sigma}^{(F)}\left(\xi_{\mathbf{k}_{2}}^{(S)}, \Delta_{\mathbf{k}_{2}}, e V\right)_{\mathrm{WKB}}=\sum_{k_{1 z}} q_{1 z} q_{2 z} g\left(\xi_{\mathbf{k}_{1}^{\prime} \sigma}^{(F)}, \xi_{\mathbf{k}_{2}}^{(S)}, \Delta_{\mathbf{k}_{2}}, e V\right)
\end{gathered}
$$


where $\mathbf{k}_{1}^{\prime} \equiv\left(k_{2 x}, k_{2 y}, k_{1 z}\right)$ and the $D_{\sigma}^{(F)}(x)$ is the total density of states (TDOS) of the ferromagnetic layer $F$ for the $\sigma$-spin state as a function of $x$.

In the present paper, one-electron energies such as $\xi_{\mathbf{k}_{1} \sigma}^{(F)}$ and $\xi_{\mathbf{k}_{2}}^{(S)}$, which are for ferromagnetic $F$ and superconducting $S$ layers, are calculated on the basis of the band theory using a universal tight-binding parameters (UTBP) method proposed by Harrison [12]. The energies of the atomic orbitals used in the band structure calculations have been calculated by using the spin-polarized self-consistent-field (SP-SCF) atomic structure calculations based on the Herman and Skillman prescription [13] using the Schwarz exchange correlation parameters [14]. The calculation procedure of the present band structure calculation is the same as that of our previous band structure calculation [15].

The energy of an electron with a $\sigma$-spin state is calculated for the wavenumber $\mathbf{k}$ within the 1st Brillouin zone (BZ) $\Omega$ and the total wave function $\Psi_{\mathbf{k} \sigma}(\mathbf{r})$ is expanded by using Bloch orbitals $\chi_{L}^{(\mu)}(\mathbf{k} \sigma, \mathbf{r})$ as follows:

$$
\Psi_{\mathbf{k} \sigma}(\mathbf{r})=\sum_{\mu} \sum_{L} \lambda_{L}^{(\mu)}(\mathbf{k} \sigma) \chi_{L}^{(\mu)}(\mathbf{k} \sigma, \mathbf{r}),
$$

where $\mu$ and $L$ are the site to be considered and the quantum state of atomic orbital, respectively, and $\lambda_{L}^{(\mu)}(\mathbf{k} \sigma)$ is the coefficient in the Bloch expansion of the total wave funtion. Therefore, the following relation is found for the superconducting layer $S$ :

$$
\left\langle\Psi_{\mathbf{k}_{2}}(\mathbf{r}) \mid \Psi_{\mathbf{k}_{2}}(\mathbf{r})\right\rangle=\sum_{\mu_{S}} \sum_{L_{S}}\left|\lambda_{L_{S}}^{\left(\mu_{S}\right)}\left(\mathbf{k}_{2}\right)\right|^{2}=1
$$

Here it is noted again that the $\mathbf{k}_{2} \sigma$ can be regarded as $\mathbf{k}_{2}$ when the CPs in superconducting layer $S$ are in the spin-singlet state. An index " $S$ " in $\mu_{S}$ and $L_{S}$ means the superconducting layer $S$, so the $\mu_{S}$ and $L_{S}$ are the site and quantum state adopted in the band structure calculation of the $S$.

Using (8), (3) can be rewritten as follows:

$$
\begin{aligned}
i_{T, \sigma}(V) & =\frac{2 \pi e}{\hbar} \widetilde{T}^{2} \sum_{\mu_{S}} \sum_{L_{S}} \eta_{\sigma} \sum_{\mathbf{k}_{2}}^{\Omega_{S}} \Theta_{\sigma}^{(F)}\left(\xi_{\mathbf{k}_{2}}^{(S)}, \Delta_{\mathbf{k}_{2}}, e V\right)\left|\lambda_{L_{S}}^{\left(\mu_{S}\right)}\left(\mathbf{k}_{2}\right)\right|^{2} \\
& \equiv \frac{2 \pi e}{\hbar} \widetilde{T}^{2} \sum_{\mu_{S}} \sum_{L_{S}} \kappa_{\sigma}^{(F)}\left(\mu_{S}, L_{S}, V\right),
\end{aligned}
$$

where $\Omega_{S}$ is the first $\mathrm{BZ}$ of $S . \eta_{\sigma}$ is defined by

$$
\eta_{\sigma}=\frac{\left|T_{\sigma}\right|^{2}}{\left|T_{\uparrow}\right|^{2}+\left|T_{\downarrow}\right|^{2}} \equiv \frac{\left|T_{\sigma}\right|^{2}}{\widetilde{T}^{2}} .
$$

The $\eta_{\sigma}$ defined here is the tunneling probability of a $\sigma$-spin electron in the F/I/S tunneling junction, so it is sure that the value of $\eta_{\sigma}$ strongly depends on the magnetic nature of an insulating layer $I$. It is clear that when the $I$ shows no magnetic nature, the tunneling probabilities of majority and minority spin electrons should be equal, that is, $\eta_{\uparrow}=\eta_{\downarrow}=$
$1 / 2$, and that when the $I$ shows magnetic nature, those should differ from each other, that is, $\eta_{\uparrow} \neq \eta_{\downarrow}$. As already stated, the present model does not explicitly include the details of the magnetic nature of the $I$; therefore, the value of $\eta_{\uparrow}\left(=1-\eta_{\downarrow}\right)$ should be treated as a parameter if we wish to extend the present model to the case in which the $I$ shows the magnetic nature. It is sure that when the magnetization of $F, \mathbf{M}_{F}$, is parallel to that of $I, \mathbf{M}_{I}$, that is, $\mathbf{M}_{F} \| \mathbf{M}_{I}$, the current due to the majority spin $I_{\mathrm{Maj}}$ is larger than the minority one $I_{\mathrm{Min}}$, and in the case of $\mathbf{M}_{F} \|-\mathbf{M}_{I}$, the $I_{\text {Maj }}$ is largely decreased as compared with the parallel case $\mathbf{M}_{F} \| \mathbf{M}_{I}$. Namely, a relation $\eta_{\uparrow}>\eta_{\downarrow}$ should be satisfied for the parallel case $\mathbf{M}_{F} \| \mathbf{M}_{I}$, and a relation $\eta_{\uparrow}<\eta_{\downarrow}$ should be satisfied for the antiparallel case $\mathbf{M}_{F} \|-\mathbf{M}_{I}$. In the present paper, therefore, $\eta_{\uparrow}\left(=1-\eta_{\downarrow}\right)$ is treated as a parameter characterizing the magnetic nature of the insulating layer $I$.

The $\kappa_{\sigma}^{(F)}\left(\mu_{S}, L_{S}, V\right)$ defined in (9) shows the partial current term due to the tunneling of a $\sigma$ spin electron. Only the band structure calculation makes it possible to evaluate the $\kappa_{\sigma}^{(F)}\left(\mu_{S}, L_{S}, V\right)$. Therefore, from the calculations of $\kappa_{\sigma}^{(F)}\left(\mu_{S}, L_{S}, V\right)$, we can directly see which atomic orbitals contribute to the F/I/S tunneling phenomena. This is just an advantage of the use of the $\kappa_{\sigma}^{(F)}\left(\mu_{S}, L_{S}, V\right)$.

In the calculation of the $I-V$ characteristics, if we write the maximum voltage $V_{\max }(>0)$ as $n_{\max } \Delta(T) / e$, that is, $e V_{\max }=$ $n_{\max } \Delta(T)$, then the calculation done for $n_{\max } \simeq 5$ is good enough to see the overall profile of the $I-V$ characteristics, since the $I-V$ curve gradually reaches to the ohmic line due to increasing the voltage $V$. On the practical point of view, it is convenient to define the normalized current $i_{T}^{( \pm)}(V)_{\text {Nor }}$. We define it as follows:

$$
\begin{aligned}
& i_{T}^{( \pm)}(V)_{\text {Nor }} \\
& \quad \equiv \frac{n_{\max } I_{T}^{( \pm)}(V)}{I_{T}^{(+)}\left(V_{\max }\right)} \\
& \quad=\sum_{\mu_{S}} \sum_{L_{S}}\left(\kappa_{\uparrow}^{(F)}\left(\mu_{S}, L_{S}, V\right)_{\text {Nor }} \pm \kappa_{\downarrow}^{(F)}\left(\mu_{S}, L_{S}, V\right)_{\text {Nor }}\right) .
\end{aligned}
$$

Here, $\kappa_{\sigma}^{(F)}\left(\mu_{S}, L_{S}, V\right)_{\text {Nor }}$ is defined by

$$
\begin{gathered}
\kappa_{\sigma}^{(F)}\left(\mu_{S}, L_{S}, V\right)_{\text {Nor }} \\
\equiv \frac{n_{\max } \kappa_{\sigma}^{(F)}\left(\mu_{S}, L_{S}, V\right)}{\sum_{\mu_{S}} \sum_{L_{S}} \sum_{\sigma} \kappa_{\sigma}^{(F)}\left(\mu_{S}, L_{S}, V_{\max }\right)} \\
\equiv \frac{n_{\max }}{K^{(F)}\left(V_{\max }\right)} \kappa_{\sigma}^{(F)}\left(\mu_{S}, L_{S}, V\right),
\end{gathered}
$$

using $\kappa_{\sigma}^{(F)}\left(\mu_{S}, L_{S}, V\right)$ defined in (9). Here note that $K^{(F)}\left(V_{\max }\right)>0$.

From the above considerations, it is concluded that, in order to understand the nature of the charge and spin transfers in the $\mathrm{F} / \mathrm{I} / \mathrm{S}$ tunneling junctions, it is necessary to calculate the $\kappa_{\sigma}^{(F)}\left(\mu_{S}, L_{S}, V\right)_{\text {Nor }}$ defined by (12), that is, $\kappa_{\sigma}^{(F)}\left(\mu_{S}, L_{S}, V\right)$ defined in (9), as correctly as possible. 
On the above considerations, we have implicitly assumed that the external magnetic field $H$ applying the junctions is not strong; that is, the Zeeman energy $\mu_{B} H$ is small as compared with the amplitude of the superconducting gap. Therefore, the effect of the magnetic field $H$ can be ignored in the present considerations. Usually, such a treatment has no problem because the value of $\mu_{B} H$ is very small; for example, that is only $5.79 \mathrm{meV}$ even in the case of the extremely strong magnetic field $H=100 \mathrm{~T}$. Exactly speaking, however, the effect of the Zeeman energy must be taken into account. In the present paper, its effect has been taken into account by using the same method done by Tedrow and Meservey [16]. Namely, the quasiparticle excitation energy $E_{\mathbf{k}}$ has been replaced by $E_{\mathbf{k}} \mp \mu_{B} H$ for the majority and the minority spin, respectively. Actually, we did a calculation for the external magnetic induction $\mathbf{B}$ with the value of $1 \mathrm{~T}$ and found that there is no detectable difference between the calculations for $\mathbf{B}=0$ and $1 \mathrm{~T}$. In the present paper, therefore, the effect of the external magnetic field has not been considered anymore.

\section{Results and Discussion}

3.1. Electronic Structures. The electronic structures of a Hg1223 high- $T_{c}$ superconductor with $\delta=0.4$, that is, $\mathrm{HgBa}_{2} \mathrm{Ca}_{2} \mathrm{Cu}_{3} \mathrm{O}_{8.4}$, and the ferromagnetic Fe with bcc structure have been calculated on the basis of the band theory using the UTBP method. In the band structure calculations, the following atomic orbitals have been used: $6 \mathrm{~s}$ and $6 \mathrm{p}$ orbits of $\mathrm{Hg}, 6 \mathrm{~s}$ and $6 \mathrm{p}$ ones of $\mathrm{Ba}, 4 \mathrm{~s}$ and $4 \mathrm{p}$ of $\mathrm{Ca}, 3 \mathrm{~d}, 4 \mathrm{~s}$, and $4 \mathrm{p}$ of $\mathrm{Cu}, 2 \mathrm{~s}$ and $2 \mathrm{p}$ of $\mathrm{O}$, and $3 \mathrm{~d}, 4 \mathrm{~s}$, and $4 \mathrm{p}$ orbits of $\mathrm{Fe}$. In order to get the electronic structure of $\mathrm{HgBa}_{2} \mathrm{Ca}_{2} \mathrm{Cu}_{3} \mathrm{O}_{8.4}$ as correctly as possible, we have calculated it by using a unit cell consisting of $2 \mathrm{Hg}, 4 \mathrm{Ba}, 4 \mathrm{Ca}, 6 \mathrm{Cu}$, and $17 \mathrm{O}$. The unit cell of $\mathrm{Hg}_{2} \mathrm{Ba}_{4} \mathrm{Ca}_{4} \mathrm{Cu}_{6} \mathrm{O}_{17}\left(=\mathrm{HgBa}_{2} \mathrm{Ca}_{2} \mathrm{Cu}_{3} \mathrm{O}_{8.5}\right)$ is shown in Figure 1, and the crystallographic data for $\mathrm{Hg}-1223$ with $\delta=0.44, \mathrm{HgBa}_{2} \mathrm{Ca}_{2} \mathrm{Cu}_{3} \mathrm{O}_{8.44}$, are tabulated in Table 1 . In the present paper, the electronic structure of $\mathrm{HgBa}_{2} \mathrm{Ca}_{2} \mathrm{Cu}_{3} \mathrm{O}_{8.4}$ has been obtained by hole-doping into the electronic structure of $\mathrm{HgBa}_{2} \mathrm{Ca}_{2} \mathrm{Cu}_{3} \mathrm{O}_{8.5}$ we have calculated. The calculated densities of states (DOSs) are shown in Figure 2. Figure 2 clearly shows that the electronic structures near the Fermi level $E_{F}$ are made from $\mathrm{Cu} 3 \mathrm{~d}$ and $\mathrm{O} 2$ p orbitals. This result allows us to suppose that the electronic structures near the $E_{F}$ come from $\mathrm{CuO}_{2}$ layers in $\mathrm{Hg}-1223$ high- $T_{c}$ superconductor. Actually, we have clearly found it from the calculated results. This is a common feature found in all the copper-oxide high$T_{c}$ superconductors.

The electron configuration used in the SP-SCF atomic structure calculation for the spin-polarized iron is $3 d_{\uparrow}^{x} 3 d_{\downarrow}^{y} 4 s_{\uparrow}^{1} 4 s_{\downarrow}^{1} 4 p_{\uparrow}^{0} 4 p_{\downarrow}^{0}$, where $x+y=6$. By using the spin-polarized self-consistent atomic data, the electronic structures of ferromagnetic $\mathrm{Fe}$ have been calculated for bcc structure. The calculated results are shown in Figures $3(\mathrm{a})$ to 3(e). Figure 3(a) is for nonmagnetic phase, that is, $(x, y)=(3,3)$, and Figures 3(b), 3(c), 3(d), and 3(e) are for $(x, y)=(3.5,2.5),(4,2),(4.5,1.5)$, and $(5,1)$. Magnetic moment $\mu / \mu_{B}$ calculated per an atom is $0.639,2.067,2.507$,
TABLE 1: Crystallographic data [8] of unit cell $\mathrm{HgBa}_{2} \mathrm{Ca}_{2} \mathrm{Cu}_{3} \mathrm{O}_{8.5}=$ $\mathrm{Hg}_{2} \mathrm{Ba}_{4} \mathrm{Ca}_{4} \mathrm{Cu}_{6} \mathrm{O}_{17}$ shown in Figure 1. Lattice constants $a(=b)$ and $c$ are 3.8502 and $31.5658 \AA$, respectively. The position $(x, y, z)$ of an atom in the unit cell is defined as $(u a, v b, w c)$, and the values of $u, v$, and $w$ are tabulated here.

\begin{tabular}{|c|c|c|c|}
\hline Atom & $u$ & $v$ & $w$ \\
\hline Hgl & 0 & 0 & 0 \\
\hline $\mathrm{Hg} 2$ & 0 & 0 & 0.5 \\
\hline Bal & 0.5 & 0.5 & 0.08865 \\
\hline $\mathrm{Ba} 2$ & 0.5 & 0.5 & 0.4113 \\
\hline $\mathrm{Ba} 3$ & 0.5 & 0.5 & 0.5887 \\
\hline $\mathrm{Ba} 4$ & 0.5 & 0.5 & 0.9113 \\
\hline Cal & 0.5 & 0.5 & 0.1977 \\
\hline $\mathrm{Ca} 2$ & 0.5 & 0.5 & 0.3023 \\
\hline $\mathrm{Ca} 3$ & 0.5 & 0.5 & 0.6977 \\
\hline $\mathrm{Ca} 4$ & 0.5 & 0.5 & 0.8023 \\
\hline $\mathrm{Cul}$ & 0 & 0 & 0.1489 \\
\hline $\mathrm{Cu} 2$ & 0 & 0 & 0.25 \\
\hline $\mathrm{Cu} 3$ & 0 & 0 & 0.3511 \\
\hline $\mathrm{Cu} 4$ & 0 & 0 & 0.6489 \\
\hline $\mathrm{Cu} 5$ & 0 & 0 & 0.75 \\
\hline $\mathrm{Cu} 6$ & 0 & 0 & 0.8511 \\
\hline $\mathrm{O} 1$ & 0 & 0 & 0.0618 \\
\hline $\mathrm{O} 2$ & 0 & 0.5 & 0.1489 \\
\hline $\mathrm{O} 3$ & 0.5 & 0 & 0.1489 \\
\hline $\mathrm{O} 4$ & 0 & 0.5 & 0.25 \\
\hline O5 & 0.5 & 0 & 0.25 \\
\hline O6 & 0 & 0.5 & 0.3511 \\
\hline O7 & 0.5 & 0 & 0.3511 \\
\hline O8 & 0 & 0 & 0.4382 \\
\hline O9 & 0.5 & 0.5 & 0.5 \\
\hline $\mathrm{O} 10$ & 0 & 0 & 0.5618 \\
\hline O11 & 0 & 0.5 & 0.6489 \\
\hline $\mathrm{O} 12$ & 0.5 & 0 & 0.6489 \\
\hline $\mathrm{O} 13$ & 0 & 0.5 & 0.75 \\
\hline $\mathrm{O} 14$ & 0.5 & 0 & 0.75 \\
\hline $\mathrm{O} 15$ & 0 & 0.5 & 0.8511 \\
\hline $\mathrm{O} 16$ & 0.5 & 0 & 0.8511 \\
\hline O17 & 0 & 0 & 0.9382 \\
\hline
\end{tabular}

and 2.900 for Figures 3(b), 3(c), 3(d), and 3(e). Here note that the measured value of $\mu$ is $2.218 \mu_{B}$ [10].

3.2. I-V Characteristics. As a tunneling process, we study here three processes such as coherent, incoherent, and WKB cases. In order to do so, we must evaluate the value of the function $\Theta_{\sigma}^{(F)}\left(\xi_{\mathbf{k}_{2}}^{(S)}, \Delta_{\mathbf{k}_{2}}, e V\right)$ in (3). The TDOS $D_{\sigma}^{(F)}(x)$ in (5) can be calculated separately; therefore, many CPU time is not required to calculate the function $\Theta_{\sigma}^{(F)}\left(\xi_{\mathbf{k}_{2}}^{(S)}, \Delta_{\mathbf{k}_{2}}, e V\right)_{\text {Inc }}$ for the incoherent case, because two band structure calculations for 

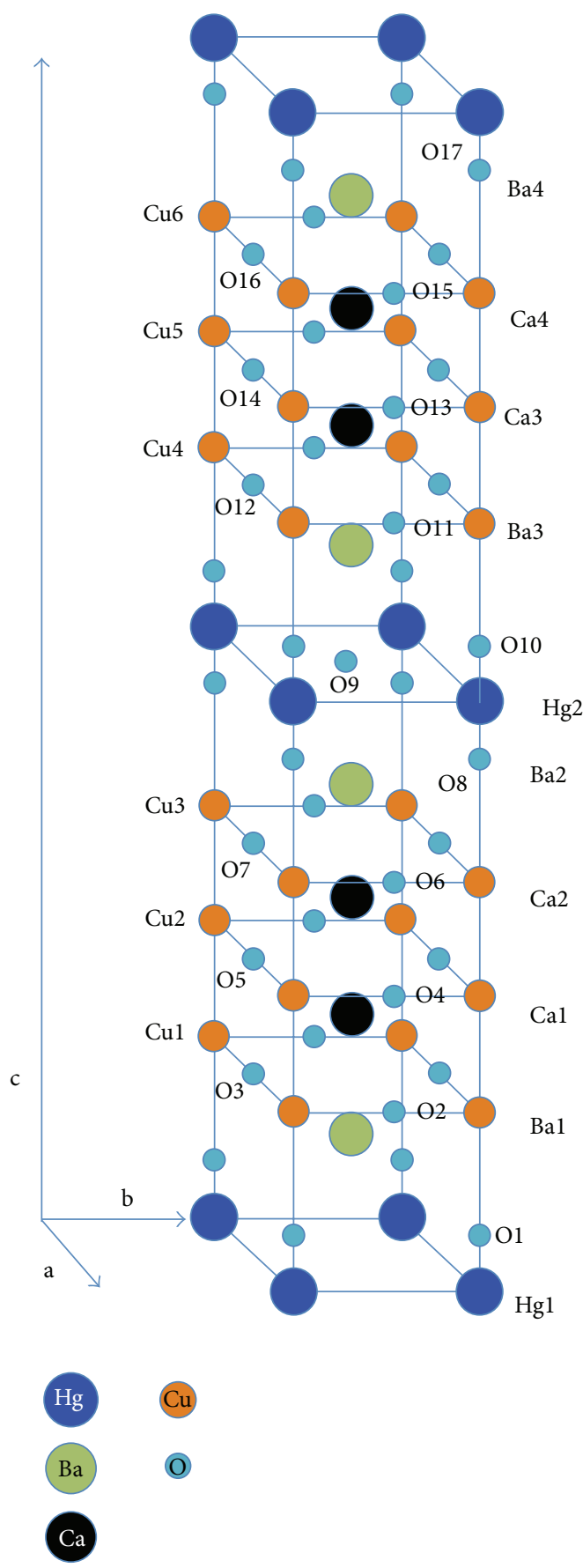

FIgure 1: Unit cell of $\mathrm{Hg}_{2} \mathrm{Ba}_{4} \mathrm{Ca}_{4} \mathrm{Cu}_{6} \mathrm{O}_{17}\left(=\mathrm{HgBa}_{2} \mathrm{Ca}_{2} \mathrm{Cu}_{3} \mathrm{O}_{8.5}\right)$ used in the band structure calculation. Lattice constants $a(=b)$ and $c$ are 3.8502 and $31.5658 \AA$, respectively.

the $S$ and $F$ layers can be done independently. In the calculations of $\Theta_{\sigma}^{(F)}\left(\xi_{\mathbf{k}_{2}}^{(S)}, \Delta_{\mathbf{k}_{2}}, e V\right)_{\mathrm{Coh}}$ and $\Theta_{\sigma}^{(F)}\left(\xi_{\mathbf{k}_{2}}^{(S)}, \Delta_{\mathbf{k}_{2}}, e V\right)_{\mathrm{WKB}}$, however, many CPU time is needed since two band structure calculations for the $S$ and $F$ are correlated with each other, as can be seen from (4) and (6). In the calculation of $\Theta_{\sigma}^{(F)}\left(\xi_{\mathbf{k}_{2}}^{(S)}, \Delta_{\mathbf{k}_{2}}, e V\right)_{\mathrm{WKB}}$, moreover, the summation over $k_{1 z}$ must be done so that a very large $\mathrm{CPU}$ time is needed for the WKB case.

The $I-V$ characteristics calculated for three cases are shown in Figure 4. Figures 4(a), 4(b), and 4(c) on the first line are results calculated for the nonmagnetic $\mathrm{Fe}$, that is, N/I/S junction, Figures 4(d), 4(e), and 4(f) on the second one are for the spin-polarized $\mathrm{Fe}$ of the electron configuration $3 d_{\uparrow}^{x} 3 d_{\downarrow}^{y}$ with $(x, y)=(4,2)$, and Figures $4(\mathrm{~g}), 4(\mathrm{~h})$, and $4(\mathrm{i})$ on the third one are for that with $(x, y)=(5,1)$. The curves drawn in Figures 4(a), 4(d), and 4(g), which are on the left column, are results calculated as the coherent tunneling, those in Figures 4(b), 4(e), and 4(h) on the central column are those as the incoherent one, and those in Figures 4(c), 4(f), and 4(i) on the right one are those as the WKB treatment. When the tunneling occurs coherently, Figures 4(a), 4(d), and $4(\mathrm{~g})$ clearly show some regions in which the differential conductance is evaluated as the negative value which is not reasonable. Namely, the present calculation tells us that the coherent tunneling does not occur in the F/I/S and N/I/S junctions considered here. It should be noted here that the accumulation number of the $\mathbf{k}_{2}$ in (3) varies as a function of the applied voltage $V$. This may be the reason why the negative differential conductance is found in the case of coherent tunneling.

In the results calculated as the incoherent and WKB cases, there is no region of the negative differential conductance, except for the region around the normalized voltage -3 in Figure 4(c) for the WKB treatment. Furthermore, it seems that the overall profiles of the $I-V$ characteristics calculated for the incoherent and $\mathrm{WKB}$ cases are similar to each other. As already stated, the numerical calculations for the WKB case need a huge CPU time. In the following, therefore, we present only the results calculated for the incoherent case, that is, the case such that $\left|T_{\mathbf{k}_{1} \mathbf{k}_{2} \sigma}\right|^{2}$ is evaluated as $\left|T_{\sigma}\right|^{2}$ depending only on the spin valuable $\sigma$.

The I-V characteristics calculated for a ferromagnetinsulator-superconductor (F/I/S) tunneling junction are shown in Figure 5. The normalized charge and spin currents, $i_{T}^{(+)}(V)_{\text {Nor }}$ and $i_{T}^{(-)}(V)_{\text {Nor }}$ defined by (11), are drawn together with the corresponding differential conductance $d I_{N} / d V_{N}$ obtained from the normalized current $I_{N}$ and the normalized voltage $V_{N}$. Here note that the $V_{N}$ is defined by $e V / \Delta(T)$ using the real voltage $V$ and the amplitude $\Delta(T)$ of $\Delta_{\mathbf{k}}$. In Figure 5, (a), (b), and (c) on the first line are results calculated for the $\mathrm{N} / \mathrm{I} / \mathrm{S}$ junction; that is, the iron is in nonmagnetic phase, (d), (e), and (f) on the second line are those for the F/I/S junction calculated by using a ferromagnetic iron with the $3 d$ electron configuration $3 d_{\uparrow}^{x} 3 d_{\downarrow}^{y}$ of $(x, y)=(3.5,2.5),(\mathrm{g}),(\mathrm{h})$, and (i) on the third line are those of $(x, y)=(4,2),(\mathrm{j}),(\mathrm{k})$, and $(\mathrm{l})$ on the fourth one are of $(x, y)=(4.5,1.5)$, and $(\mathrm{m}),(\mathrm{n})$, and (o) on the bottom line are those of $(x, y)=(5,1)$. The $I-V$ characteristics drawn in (a), (d), (g), (j), and (m) on the left column have been obtained by using $\left(\eta_{\uparrow}, \eta_{\downarrow}\right)=(3 / 4,1 / 4)$, those in (b), (e), (h), (k), and (n) on the middle column are for $\left(\eta_{\uparrow}, \eta_{\downarrow}\right)=(1 / 2,1 / 2)$, and those in (c), (f), (i), (l), and (o) on the right column are for $\left(\eta_{\uparrow}, \eta_{\downarrow}\right)=(1 / 4,3 / 4)$. 


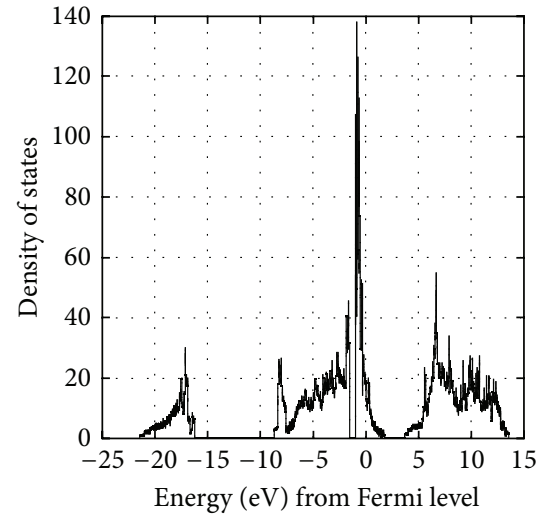

(a)

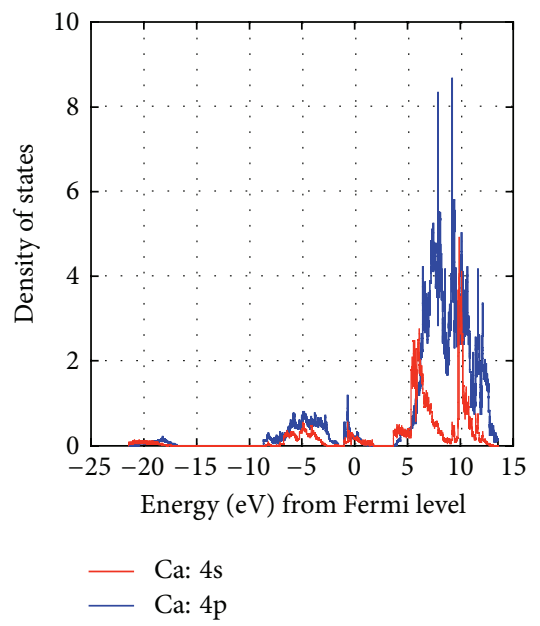

(d)

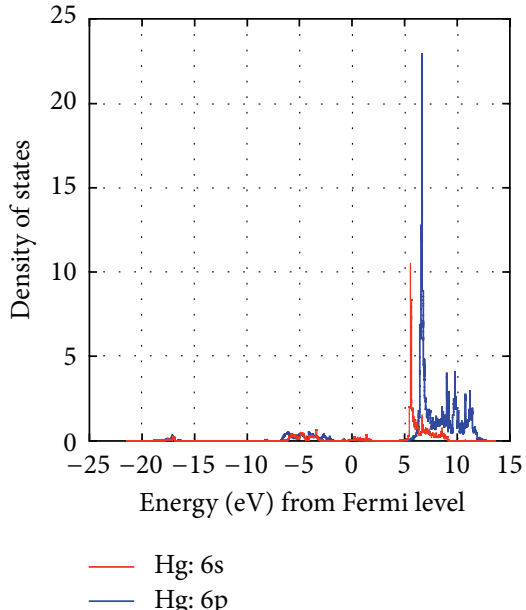

(b)
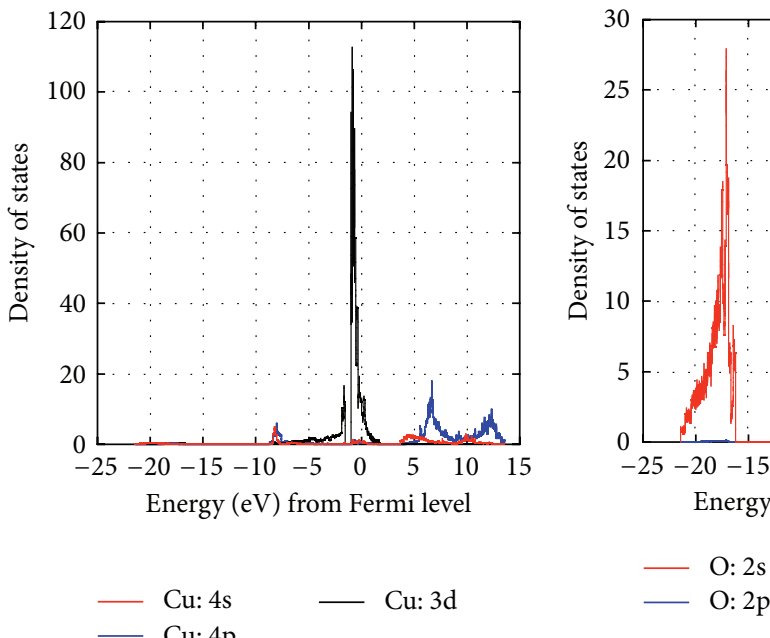

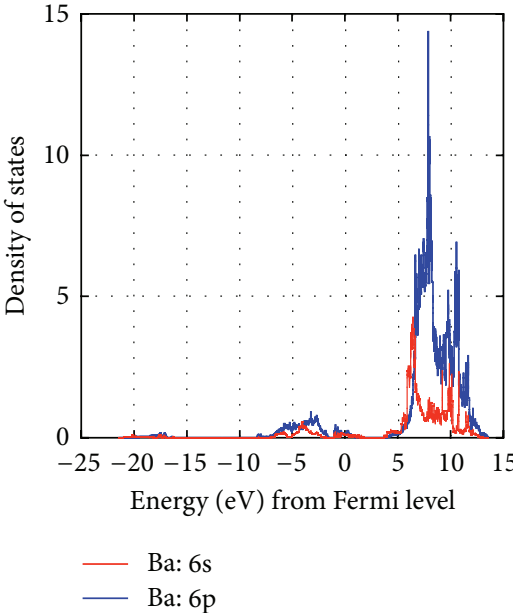

(c)

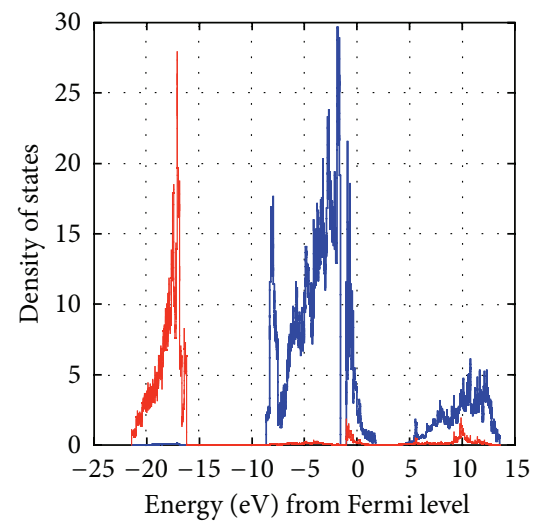

: $2 \mathrm{~s}$

(f)

Figure 2: Density of states (DOS) (states/eV · unit cell) calculated for the high- $T_{c}$ superconductor $\mathrm{HgBa}_{2} \mathrm{Ca}_{2} \mathrm{Cu}_{3} \mathrm{O}_{8.4}$. Energy (eV) is measured from Fermi level $E_{F}$. Here note that the electronic structure of $\mathrm{HgBa}_{2} \mathrm{Ca}_{2} \mathrm{Cu}_{3} \mathrm{O}_{8.4}$ is obtained by hole-doping into that of $\mathrm{HgBa}_{2} \mathrm{Ca}_{2} \mathrm{Cu}_{3} \mathrm{O}_{8.5}$. (a) is the total density of states (TDOS). (b), (c), (d), (e), and (f) are sum of partial density of states (PDOS) of respective atoms, namely, those are that of $2 \mathrm{Hg}, 4 \mathrm{Ba}, 4 \mathrm{Ca}, 6 \mathrm{Cu}$ and $17 \mathrm{O}$ atoms, respectively. For example, the DOS of $\mathrm{Cu}$ : $3 \mathrm{~d}$ drawn in (e) has been obtained by adding the $\mathrm{Cu}$ : 3d PDOSs of six $\mathrm{Cu}$ atoms ( $\mathrm{Cul}$ to $\mathrm{Cu} 6)$ shown in Figure 1 . Here note that the $\mathrm{Cu}$ : $3 \mathrm{~d}$ PDOS of the respective $\mathrm{Cu}$ atom is made by adding the five PDOSs for Cu $3 \mathrm{~d}_{x y}, 3 \mathrm{~d}_{y z}, 3 \mathrm{~d}_{z x}, 3 \mathrm{~d}_{\left\{x^{2}-y^{2}\right\}}$ and $3 \mathrm{~d}_{\left\{3 z^{2}-r^{2}\right\}}$ orbitals. The present figure clearly shows that the states near the $E_{F}$ are made from $\mathrm{Cu} 3 \mathrm{~d}$ and $\mathrm{O} 2 \mathrm{p}$ orbitals shown in (e) and (f).

The normalized current $i_{T}^{( \pm)}(V)_{\text {Nor }}$ defined by (11) can be rewritten as follows:

$$
\begin{aligned}
& i_{T}^{( \pm)}(V)_{\text {Nor }} \\
& =\frac{n_{\max }}{K^{(F)}\left(V_{\max }\right)} \sum_{\mu_{S}} \sum_{L_{S}} \sum_{\mathbf{k}_{2}}\left|\lambda_{L_{S}}^{\left(\mu_{S}\right)}\left(\mathbf{k}_{2}\right)\right|^{2} \\
& \quad \times\left(\eta_{\uparrow} \Theta_{\uparrow}^{(F)}\left(\xi_{\mathbf{k}_{2}}^{(S)}, \Delta_{\mathbf{k}_{2}}, e V\right) \pm \eta_{\downarrow} \Theta_{\downarrow}^{(F)}\left(\xi_{\mathbf{k}_{2}}^{(S)}, \Delta_{\mathbf{k}_{2}}, e V\right)\right) .
\end{aligned}
$$

Here, $\Theta_{\sigma}^{(F)}\left(\xi_{\mathbf{k}}^{(S)}, \Delta_{\mathbf{k}}, e V\right)_{\text {Inc }}$ for the incoherent case defined by (5) can be well approximated as follows:

$$
\begin{aligned}
& \Theta_{\sigma}^{(F)}\left(\xi_{\mathbf{k}}^{(S)}, \Delta_{\mathbf{k}}, e V\right)_{\text {Inc }}
\end{aligned}
$$

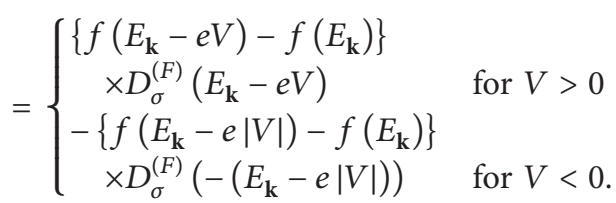

Equation (14) tells us that only the quasiparticle excitation energy $E_{\mathbf{k}}$ with the energy region of $0 \leq E_{\mathbf{k}} \leq e|V|$ is valid 


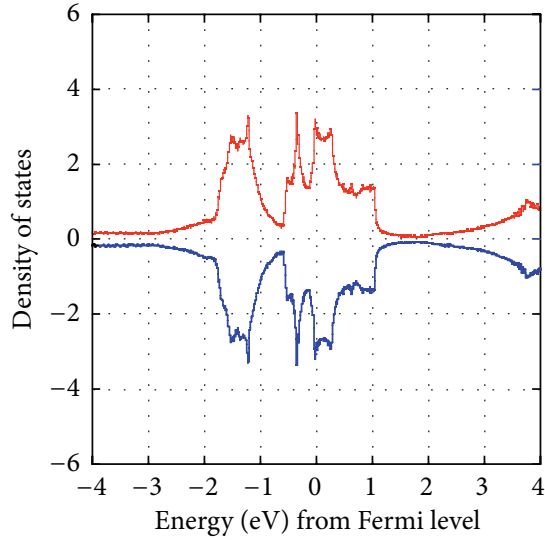

(a)

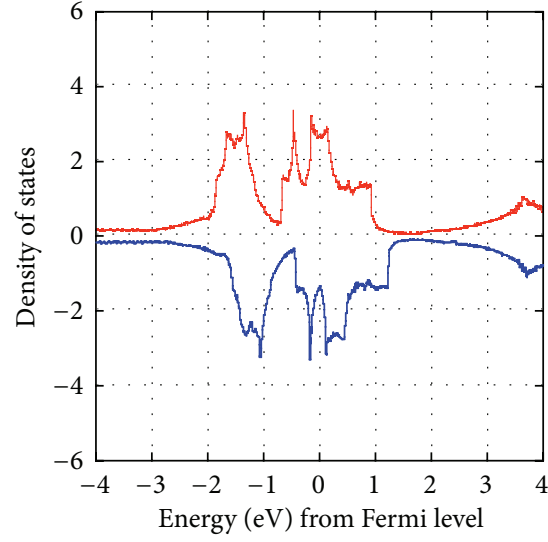

(b)

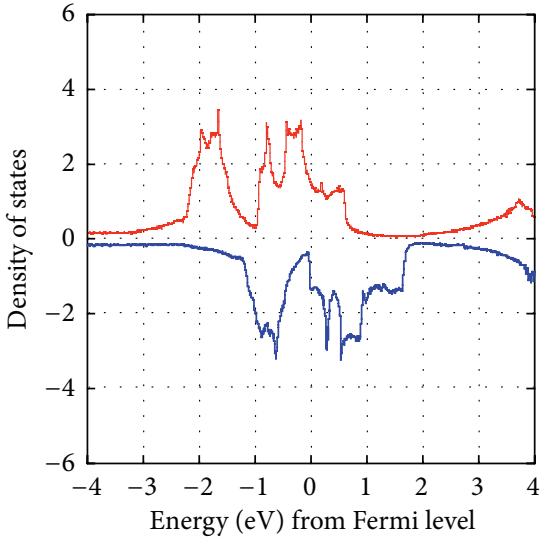

(c)

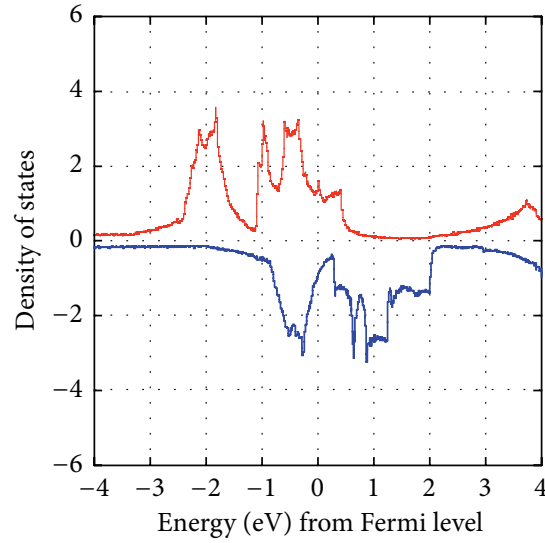

(d)

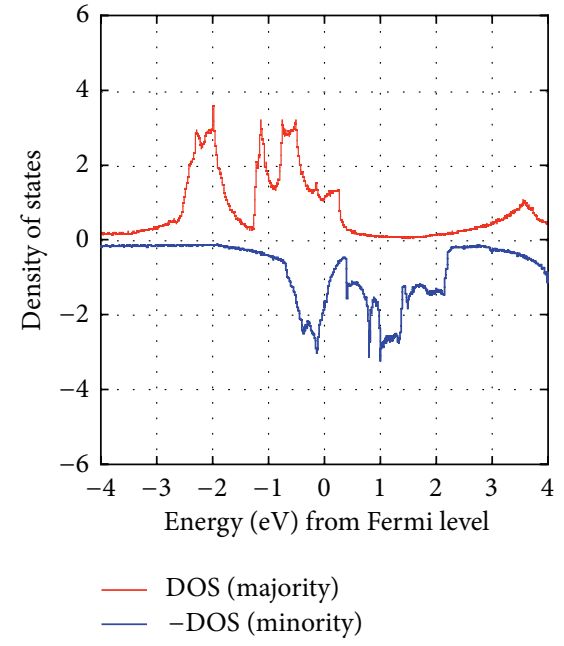

(e)

FIgURE 3: TDOSs (states/eV-atom) calculated for bcc Fe. The electron configuration used in the SP-SCF atomic structure calculation is $3 d_{\uparrow}^{x} 3 d_{\downarrow}^{y} 4 s_{\uparrow}^{1} 4 s_{\downarrow}^{1} 4 p_{\uparrow}^{0} 4 p_{\downarrow}^{0}$, where $x+y=6$. (a), (b), (c), (d), and (e) are the results calculated for $(x, y)=(3,3),(3.5,2.5),(4,2),(4.5,1.5)$, and $(5,1)$. The values of DOS $D_{\sigma}^{(F)}(0)$ at $E_{F}$ are follows: $\left(D_{\uparrow}^{(F)}(0), D_{\downarrow}^{(F)}(0)\right)=(2.829,2.829),(2.669,1.403),(1.533,1.355),(1.368,0.921)$, and $(1.091,1.743)$ for (a), (b), (c), (d), and (e), respectively. Here note that a relation $D_{\uparrow}^{(F)}(0)>D_{\downarrow}^{(F)}(0)$ is satisfied except for (e). Magnetic moment $\mu / \mu_{B}$ calculated per an atom is $0.639,2.067,2.507$, and 2.900 for (b), (c), (d), and (e), respectively. The calculated value should be compared with the experimental one $2.218 \mu_{B}[10]$.

for the evaluations of tunneling currents. Therefore, the DOS $D_{\sigma}^{(F)}(\rho)$ as a function of the energy $\rho$ is evaluated only in the energy region of $|\rho| \leq e|V|$. From a relation $e|V| \leq n_{\max } \Delta(T)$ and our calculation conditions $n_{\max }=5$ and $\Delta\left(T_{\mathrm{LN}}\right)=$ $70 \mathrm{meV}$, the DOS $D_{\sigma}^{(F)}(\rho)$ of ferromagnetic iron is evaluated only in the energy region of $|\rho| \leq 350 \mathrm{meV}$, whose energy is measured from the $E_{F}$.

3.2.1. Case of $\eta_{\uparrow}=\eta_{\downarrow}$. As already stated, the present case corresponds to the case when the insulating layer $I$ shows no magnetic nature, that is, the case of a standard F/I/S junction. From (b), (e), (h), (k), and (n) on the middle column of Figure 5, in which a relation $\eta_{\uparrow}=\eta_{\downarrow}=1 / 2$ is satisfied, we can see that the $I-V$ curve, the resultant differential conductance, and the spin current are changed due to the change of $\mathbf{M}_{F}$. For the spin current, moreover, an interesting result such that the minority spin current exceeds the majority one is found. This interesting result is clearly found in the positive voltage region of Figure 5(n).

On the positive voltage region, the normalized spin current $i_{T}^{(-)}(V)_{\text {Nor }}$ can be represented as

$$
\begin{aligned}
i_{T}^{(-)}(V)_{\text {Nor }}= & \frac{n_{\max }}{K^{(F)}\left(V_{\max }\right)} \sum_{\mu_{S}} \sum_{L_{S}} \sum_{\mathbf{k}_{2}}\left|\lambda_{L_{S}}^{\left(\mu_{S}\right)}\left(\mathbf{k}_{2}\right)\right|^{2} \\
& \times\left\{f\left(E_{\mathbf{k}_{2}}-e V\right)-f\left(E_{\mathbf{k}_{2}}\right)\right\} \\
& \times \frac{1}{2}\left(D_{\uparrow}^{(F)}\left(E_{\mathbf{k}_{2}}-e V\right)-D_{\downarrow}^{(F)}\left(E_{\mathbf{k}_{2}}-e V\right)\right) .
\end{aligned}
$$

Equation (15) clearly tells us that the interesting result shown in Figure 5(n) originated from the difference between the 


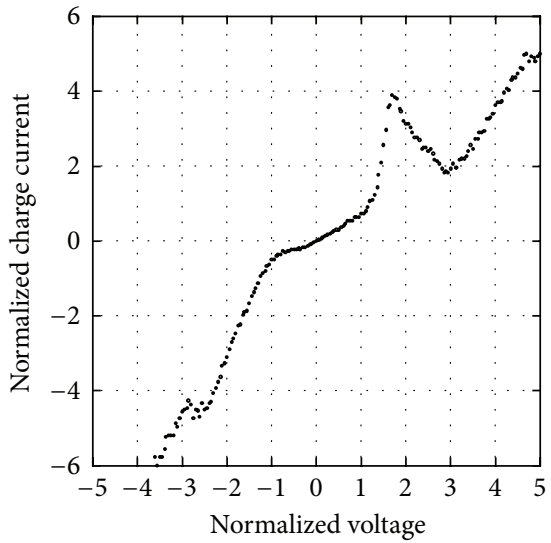

(a)

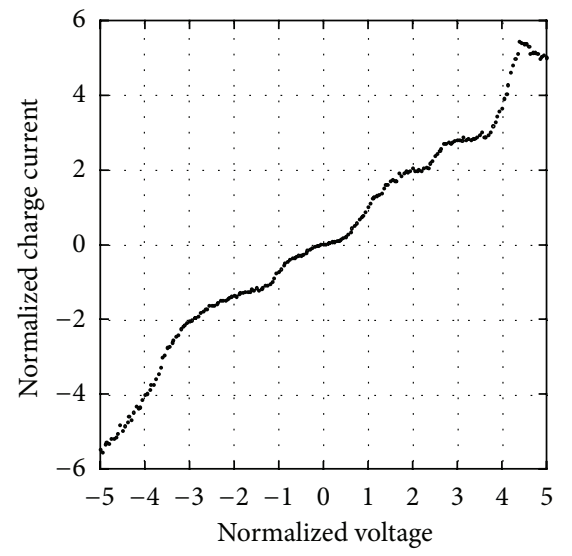

(d)

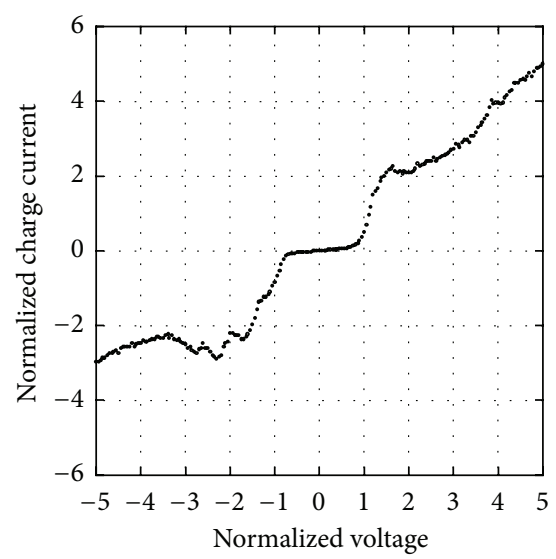

(g)

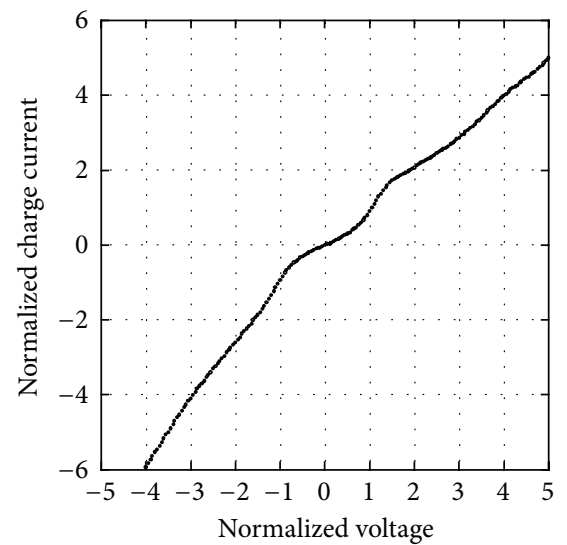

(b)

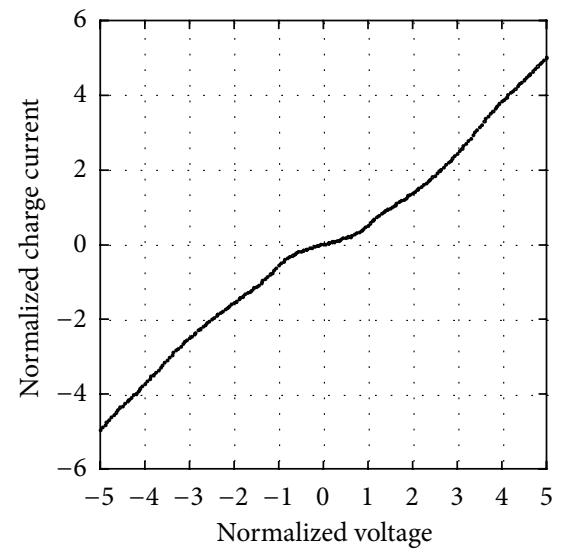

(e)

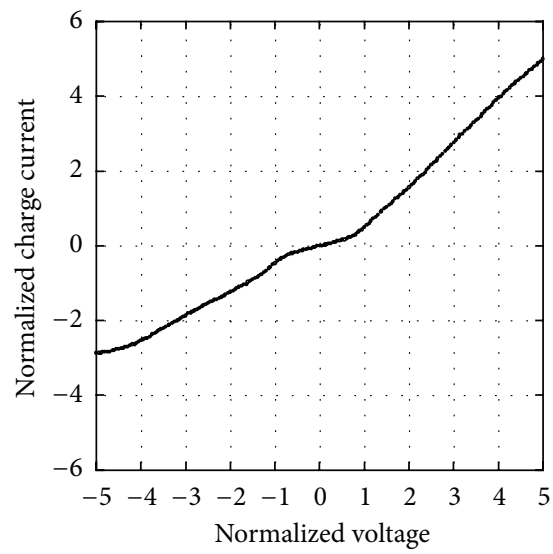

(h)

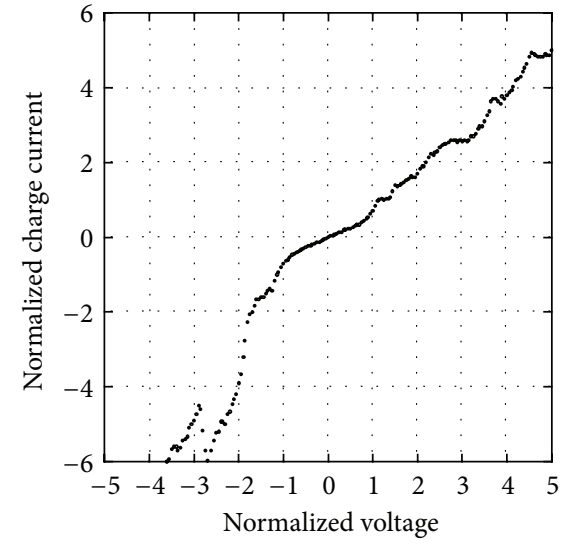

(c)

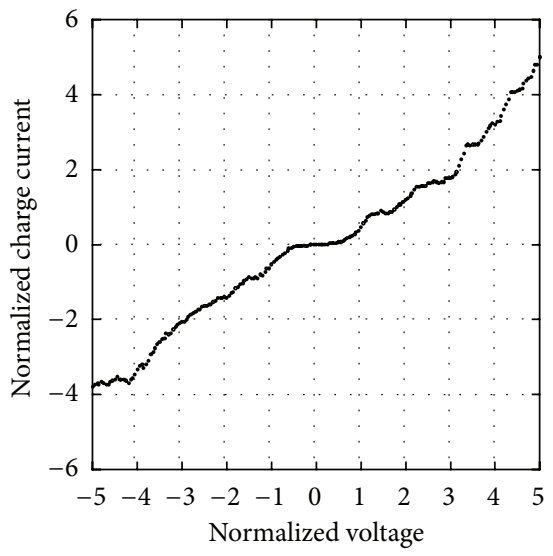

(f)

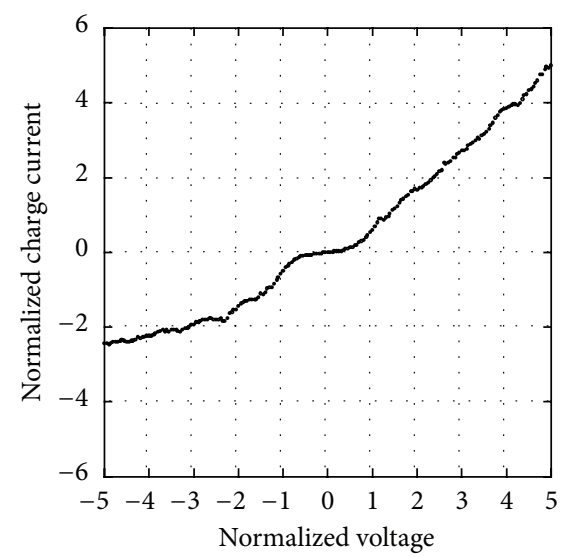

(i)

FIGURE 4: $I-V$ characteristics calculated for the ferromagnet-insulator-superconductor (F/I/S) tunneling junctions. Ferromagnet is an iron with bcc structure and the superconductor is $\mathrm{Hg}-1223$ high- $T_{c}$ superconductor $\mathrm{HgBa}_{2} \mathrm{Ca}_{2} \mathrm{Cu}_{3} \mathrm{O}_{8.4}$. The normalized charge current $i_{T}^{(+)}(V)_{\mathrm{Nor}}$ is defined by (11), and the normalized voltage $V_{N}$ is defined by $\mathrm{eV} / \Delta(T)$. (a), (b), and (c) on the first line are results calculated for the nonmagnetic phase, (d), (e), and (f) on the second line are for the electron configuration $3 d_{\uparrow}^{x} 3 d^{y}$ with $(x, y)=(4,2)$, and (g), (h), and (i) on the third one are for that with $(x, y)=(5,1)$. (a), (d), and (g) on the left column are results calculated for the case of coherent tunneling, (b), (e), and (h) on the central one are for the case of incoherent tunneling, and (c), (f), and (i) on the right column are for the case of WKB treatment. In (a), (d), and $(\mathrm{g})$ calculated as the coherent tunneling, there are some regions in which the differential conductance is calculated as the negative value which is unreasonable. Moreover, also in (c) for the WKB case, the region of the negative differential conductance is found in the normalized voltage around -3 . Note that all the calculations have been done for $\eta_{\uparrow}=\eta_{\downarrow}=1 / 2$. 
DOSs of the majority and minority spin bands of ferromagnetic iron. The calculated results shown in Figure 3 show that the values of $D_{\uparrow}^{(F)}(0)$ and $D_{\downarrow}^{(F)}(0)$ satisfy the condition of $D_{\uparrow}^{(F)}(0)>D_{\downarrow}^{(F)}(0)$ except for the case shown in Figure 3(e). Here note that the results shown in Figures $5(\mathrm{~m}), 5(\mathrm{n})$, and 5(o) have been calculated by using the DOS shown in Figure 3(e). We can say that the condition $D_{\uparrow}^{(F)}(0)<D_{\downarrow}^{(F)}(0)$ has led to an interesting result mentioned above.

3.2.2. Case of $\eta_{\uparrow} \neq \eta_{\downarrow}$. Present case corresponds to the case such that the insulating layer $I$ shows a magnetic nature such as a detectable magnetization $\mathbf{M}_{I}$. As already stated, a relation $\eta_{\uparrow}>\eta_{\downarrow}$ corresponds to the $\mathbf{M}_{F} \| \mathbf{M}_{I}$, and the $\eta_{\uparrow}<$ $\eta_{\downarrow}$ does to the $\mathbf{M}_{F} \|-\mathbf{M}_{I}$. In the FIS tunneling junction with the magnetic insulating barrier, it may be expected that a proximity effect could be found between the magnetic barrier and the superconducting layer. In the present study, however, all possible proximity effects have been neglected for simplicity.

The $I-V$ characteristics calculated for the case of $\left(\eta_{\uparrow}, \eta_{\downarrow}\right)=$ $(3 / 4,1 / 4)$ are shown in $(\mathrm{a}),(\mathrm{d}),(\mathrm{g}),(\mathrm{j})$, and $(\mathrm{m})$ on the left column, and those for $\left(\eta_{\uparrow}, \eta_{\downarrow}\right)=(1 / 4,3 / 4)$ are in (c), (f), (i), (l), and (o) on the right column of Figure 5. In the present subsection, we consider the case of $\eta_{\uparrow} \neq \eta_{\downarrow}$. Therefore, in the following, only the case of the F/I/S tunneling junction, that is, the case excepting Figures 5(a), 5(b), and 5(c) for the N/I/S junction, is considered.

From (13) and (14), we can see that (I) the condition $\eta_{\uparrow}>\eta_{\downarrow}$ works by increasing the DOS $D_{\uparrow}^{(F)}(0)$ and decreasing the $D_{\downarrow}^{(F)}(0)$ and (II) the condition $\eta_{\uparrow}<\eta_{\downarrow}$ inversely works as mentioned in (I), that is, by decreasing the majority spin band and increasing the minority one. A result such that the minority spin current exceeds the majority one is clearly found in Figures 5(f), 5(1), and 5(o). We can see that the $I-V$ curve and the resultant diffrential conductance nearly remain the same as the change of $\eta_{\uparrow}\left(=1-\eta_{\downarrow}\right)$, but the spin current is largely changed due to the change of $\eta_{\uparrow}$. Such a behavior is clearly found from the comparison of three Figures 5(d), 5(e), and 5(f) on the second line. In the next subsection, therefore, we consider the spin currents found in Figures 5(d), 5(e), and $5(f)$.

3.2.3. Spin Current. It has already been stated that the $\kappa_{\sigma}^{(F)}\left(\mu_{S}, L_{S}, V\right)$ defined in (9) shows the partial current term due to the tunneling of a $\sigma$ spin electron, so that the present calculation is able to show which atomic orbitals contribute to the F/I/S tunneling phenomena. This is just an advantage of our calculation method. It is easy to divide the spin currents drawn in Figures 5(d), 5(e), and 5(f) into the atomic orbital parts, $s, p_{x}, p_{y}, p_{z}, d_{x y}, d_{y z}, d_{z x}, d_{x^{2}-y^{2}}$, and $d_{3 z^{2}-r^{2}}$. The calculated result clearly showed that the most important atomic parts are $3 d$ orbitals of $\mathrm{Cu}$, as is expected. The $\mathrm{d}_{x y}$, $d_{y z}, d_{z x}, d_{x^{2}-y^{2}}$, and $d_{3 z^{2}-r^{2}}$ parts of the spin currents drawn in Figures 5(d), 5(e), and 5(f) are shown in Figures 6(a), 6(b), and $6(\mathrm{c})$. The calculated results tell us that the most dominant spin current is observed in an electron tunneling between the
$3 d$ orbits of ferromagnetic Fe and the $\mathrm{Cu} 3 d_{x^{2}-y^{2}}$ orbit of Hg1223 high- $T_{c}$ superconductor. $\mathrm{Cu} 3 d_{x^{2}-y^{2}}$ and $\mathrm{O} 2 p_{x}$ and $2 p_{y}$ atomic orbitals make a $p d \sigma$-bonding state which acts as a stage of an electron transfer within $\mathrm{a} \mathrm{CuO}_{2}$ superconducting layer. This is a common feature found in all the copper-oxide high- $T_{c}$ superconductors; therefore, it seems that the change of the spin state of the $\mathrm{Cu} 3 d_{x^{2}-y^{2}}$ atomic orbital makes a large effort to the superconductivity. In the following, therefore, we focus our attention on only the $\mathrm{Cu} 3 d_{x^{2}-y_{2}}$ atomic orbital and consider how the change of spin should be observed.

It is well known that the experiment of the X-ray magnetic circular dichroism (XMCD) is a powerful tool to see directly the nature of the spin dependent electron state near the $E_{F}$. Now, let us consider two absorption processes such as the $\left|2 p_{1 / 2}\right\rangle \rightarrow\left|3 d_{x^{2}-y^{2}}\right\rangle$ and the $\left|2 p_{3 / 2}\right\rangle \rightarrow\left|3 d_{x^{2}-y^{2}}\right\rangle$ for the $\mathrm{Cu}$ atom in $\mathrm{Hg}-1223 \mathrm{high}-\mathrm{T}_{c}$ superconductor. The $\mathrm{Cu} 3 d_{x^{2}-y^{2}}$ orbit $\left|3 d_{x^{2}-y^{2}}\right\rangle$ is written as

$$
\left|3 d_{x^{2}-y^{2}}\right\rangle=R_{3 d}(r) \sqrt{\frac{1}{2}}\left(Y_{2}^{2}(\widehat{\mathbf{r}})+Y_{2}^{-2}(\widehat{\mathbf{r}})\right),
$$

where $R_{3 d}(r)$ is the radial wave function of $\mathrm{Cu} 3 d$ atomic orbital and $Y_{\ell}^{m}(\widehat{\mathbf{r}})$ is the spherical harmonics. Therefore, the hole state $\left|3 d_{x^{2}-y^{2}}^{\text {(hole) }}\right\rangle$ of $\left|3 d_{x^{2}-y^{2}}\right\rangle$, which is the electron state just above the $E_{F}$, can be written as

$$
\left|3 d_{x^{2}-y^{2}}^{\text {(hole) }}\right\rangle=(a|\alpha\rangle+b|\beta\rangle)\left|3 d_{x^{2}-y^{2}}\right\rangle,
$$

where $|\alpha\rangle \equiv|\uparrow\rangle,|\beta\rangle \equiv|\downarrow\rangle$, and a relation $|a|^{2}+|b|^{2}=1$ is satisfied. Using Clebsch-Gordan coefficients, the $\left|2 p_{1 / 2}\right\rangle$ and $\left|2 p_{3 / 2}\right\rangle$ states can be written as follows:

$$
\begin{aligned}
& \left|2 p_{1 / 2}\right\rangle \\
& =R_{2 p}(r) \\
& \quad \times\left\{\begin{array}{l}
-\sqrt{\frac{1}{3}} Y_{1}^{0}(\widehat{\mathbf{r}})|\alpha\rangle+\sqrt{\frac{2}{3}} Y_{1}^{1}(\widehat{\mathbf{r}})|\beta\rangle \quad\left(=\left|\frac{1}{2}, \frac{1}{2}\right\rangle\right) \\
-\sqrt{\frac{2}{3}} Y_{1}^{-1}(\widehat{\mathbf{r}})|\alpha\rangle+\sqrt{\frac{1}{3}} Y_{1}^{0}(\widehat{\mathbf{r}})|\beta\rangle
\end{array} \quad\left(=\left|\frac{1}{2},-\frac{1}{2}\right\rangle\right),\right.
\end{aligned}
$$

$$
\begin{aligned}
& \left|2 p_{3 / 2}\right\rangle \\
& =R_{2 p}(r) \\
& \times \begin{cases}Y_{1}^{1}(\widehat{\mathbf{r}})|\alpha\rangle & \left(=\left|\frac{3}{2}, \frac{3}{2}\right\rangle\right) \\
\sqrt{\frac{2}{3}} Y_{1}^{0}(\widehat{\mathbf{r}})|\alpha\rangle+\sqrt{\frac{1}{3}} Y_{1}^{1}(\widehat{\mathbf{r}})|\beta\rangle & \left(=\left|\frac{3}{2}, \frac{1}{2}\right\rangle\right) \\
\sqrt{\frac{1}{3}} Y_{1}^{-1}(\widehat{\mathbf{r}})|\alpha\rangle+\sqrt{\frac{2}{3}} Y_{1}^{0}(\widehat{\mathbf{r}})|\beta\rangle & \left(=\left|\frac{3}{2},-\frac{1}{2}\right\rangle\right) \\
Y_{1}^{-1}(\widehat{\mathbf{r}})|\beta\rangle & \left(=\left|\frac{3}{2},-\frac{3}{2}\right\rangle\right) .\end{cases}
\end{aligned}
$$




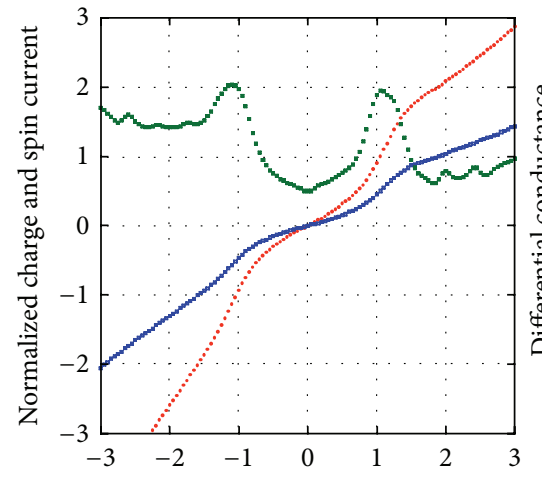

(a)

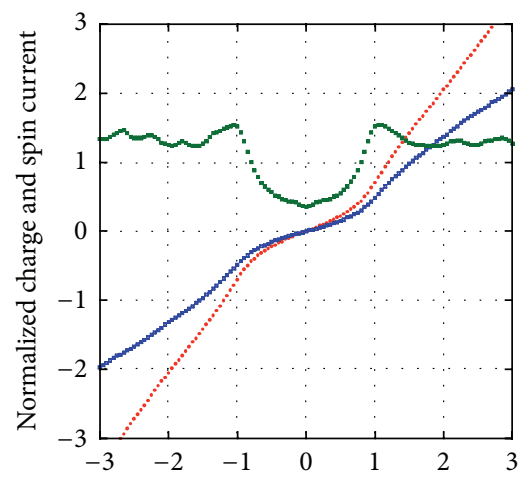

(d)

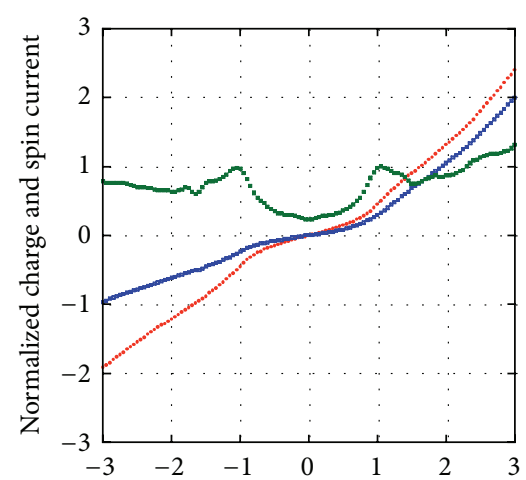

(g)

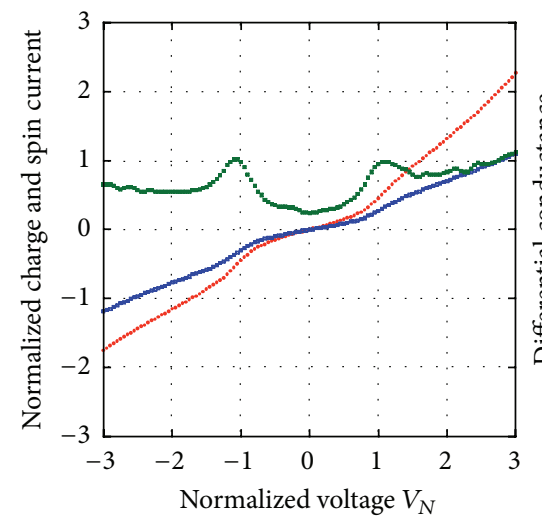

- Charge-T_norm " $\mathrm{d} l / \mathrm{dV}$
$=$ Spin-T_norm

(j)

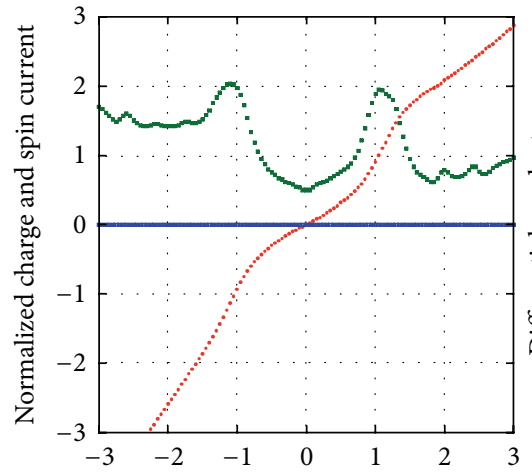

(b)

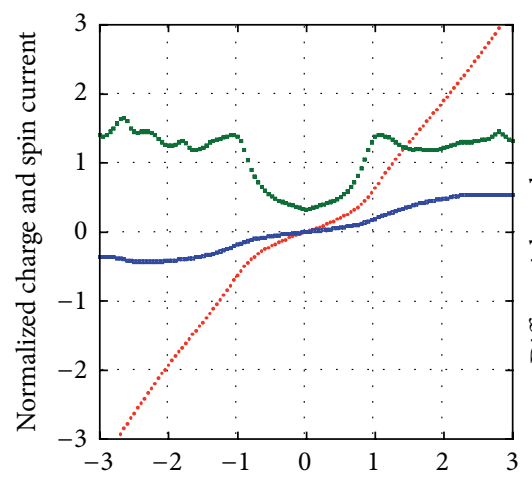

(e)

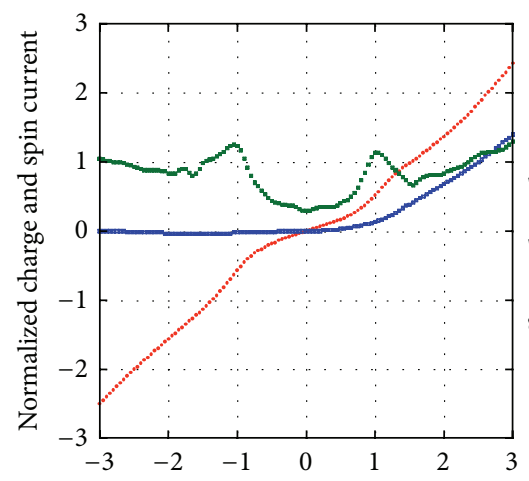

(h)

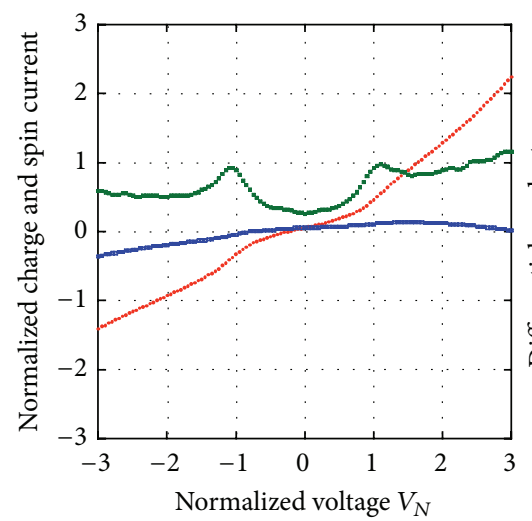

- Charge-T_norm " dl/dV - Spin-T_norm

(k)

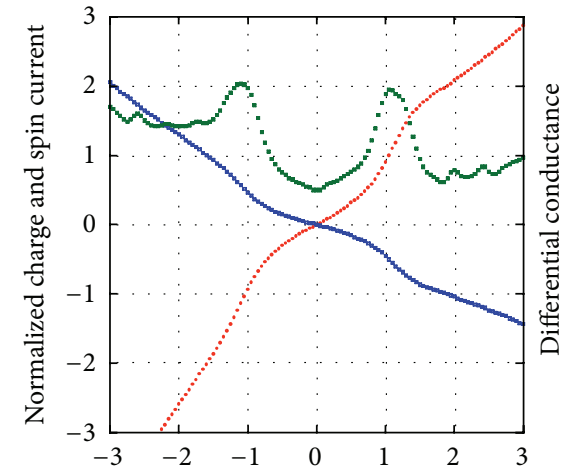

(c)

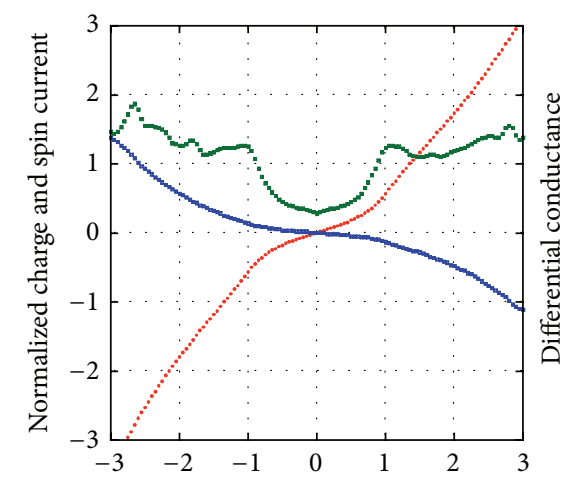

(f)

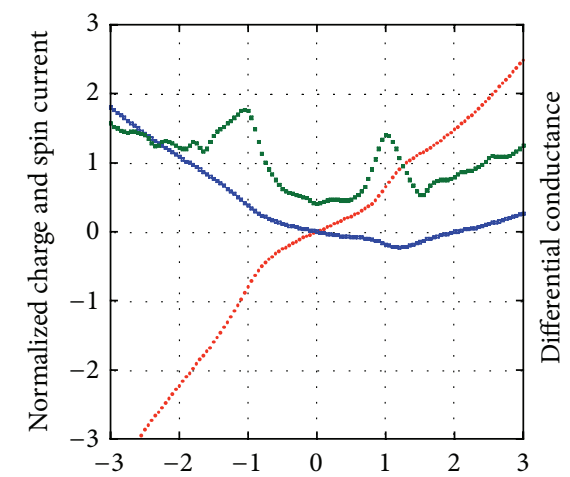

(i)

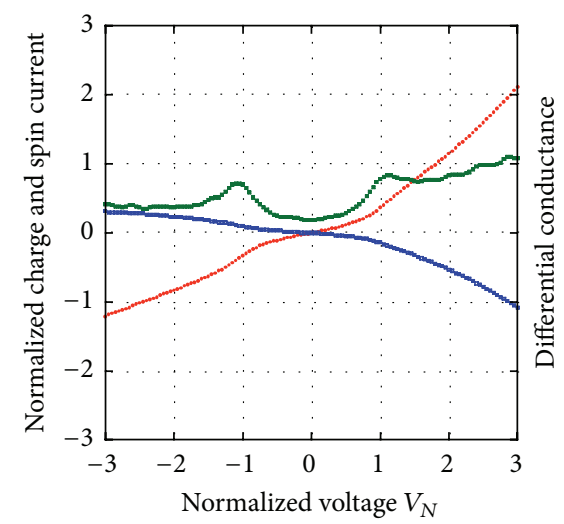

- Charge-T_norm " dl/dV " Spin-T_norm

(1)

FIgure 5: Continued. 


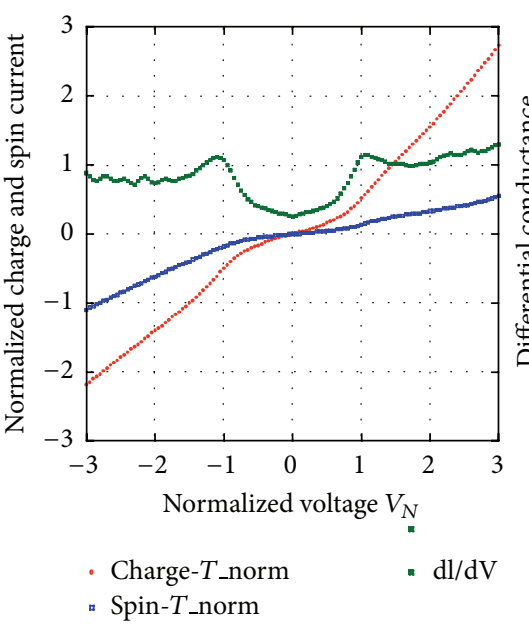

$(\mathrm{m})$

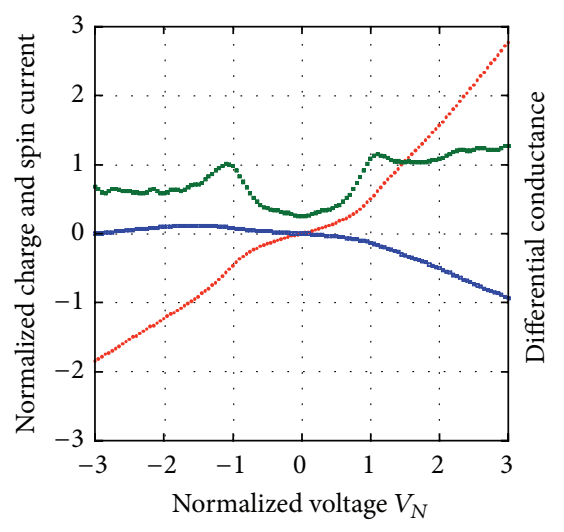

- Charge-T_norm " $\mathrm{dl} / \mathrm{dV}$
"Spin-T_norm

(n)

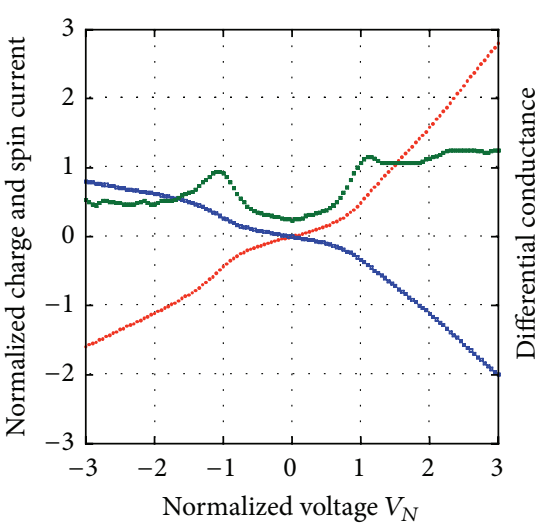

- Charge-T_norm " $\mathrm{dl} / \mathrm{dV}$ "Spin-T_norm

(o)

Figure 5: (a), (b), and (c) on the first line are results calculated for the N/I/S junction; that is, the iron is in nonmagnetic phase, whose DOS is shown in Figure 3(a). (d) to (o) are for the F/I/S junction. (d), (e), and (f) on the second line are results calculated for a ferromagnetic iron with the electron configuration $3 d_{\uparrow}^{x} 3 d^{y}$ of $(x, y)=(3.5,2.5)$, whose DOS is shown in Figure $3(\mathrm{~b}) .(\mathrm{g})$, (h), and (i) on the third line are those for $(x, y)=(4,2)$, whose DOS is shown in Figure $3(\mathrm{c}),(\mathrm{j}),(\mathrm{k})$, and $(\mathrm{l})$ on the fourth one are for $(x, y)=(4.5,1.5)$, whose DOS is shown in Figure 3(d), and (m), (n), and (o) on the bottom line are for $(x, y)=(5,1)$, whose DOS is shown in 3(e). The $I$ - $V$ characteristics drawn in (a), $(\mathrm{d}),(\mathrm{g}),(\mathrm{j})$, and $(\mathrm{m})$ on the left column have been obtained by setting a parameter $\eta_{\uparrow}\left(=1-\eta_{\downarrow}\right)$ to $3 / 4$, those in (b), (e), (h), (k), and (n) on the middle column are for $\eta_{\uparrow}=1 / 2$, and those in (c), (f), (i), (l), and (o) on the right column are for $\eta_{\uparrow}=1 / 4$.

For the circular polarized lights with the helicity $h= \pm 1$, the Hamiltonian $\widehat{H}^{\prime}$ of the dipole-allowed transition is written as

$$
\widehat{H}^{\prime}=e E \sqrt{\frac{1}{2}}(x \pm i y)=\mp e E r \sqrt{\frac{4 \pi}{3}} Y_{1}^{ \pm 1}(\widehat{\mathbf{r}}),
$$

where $E$ is the amplitude of the electric field $\mathbf{E}$. It is clear that (20) becomes a standard Hamiltonian $e E r \cos \theta$ for the dipole-allowed transition when a zero helicity light $h=0$ is considered, that is, when $Y_{1}^{ \pm 1}(\widehat{\mathbf{r}})$ is replaced by $Y_{1}^{0}(\widehat{\mathbf{r}})$. There are some allowed-absorption processes. In the present paper, only the allowed transition with $h=1$ is considered for simplicity.

It is easy to calculate the oscillator strength $\left|\left\langle 3 d_{x^{2}-y^{2}}^{(\text {(hole) }}\left|\widehat{H}^{\prime}\right| j, m_{j}\right\rangle\right|^{2}(\equiv \Gamma)$. When $h=1$, the $\Gamma$ is evaluated as follows:

$$
\Gamma=A \mid\left(a ^ { * } \left\langle\left.\alpha\left|+b^{*}\langle\beta|\right)\left\langle Y_{2}^{2}\left|Y_{1}^{1}\right| j, m_{j}\right\rangle\right|^{2},\right.\right.
$$

where $A \equiv(2 \pi / 3) e^{2} E^{2} I_{2 p \rightarrow 3 d}^{2}$ and $I_{2 p \rightarrow 3 d} \equiv$ $\int_{0}^{\infty} R_{3 d}(r) R_{2 p}(r) r^{3} d r$. An integral $\left\langle Y_{2}^{2}\left|Y_{1}^{1}\right| j, m_{j}\right\rangle$ in (21) is calculated as follows:

$$
\begin{aligned}
& \left\langle Y_{2}^{2}\left|Y_{1}^{1}\right| j, m_{j}\right\rangle \\
& = \begin{cases}\sqrt{\frac{2}{3}}\left\langle Y_{2}^{2}\left|Y_{1}^{1}\right| Y_{1}^{1}\right\rangle|\beta\rangle & \text { for }\left|\frac{1}{2}, \frac{1}{2}\right\rangle \\
\left\langle Y_{2}^{2}\left|Y_{1}^{1}\right| Y_{1}^{1}\right\rangle|\alpha\rangle & \text { for }\left|\frac{3}{2}, \frac{3}{2}\right\rangle \\
\sqrt{\frac{1}{3}}\left\langle Y_{2}^{2}\left|Y_{1}^{1}\right| Y_{1}^{1}\right\rangle|\beta\rangle & \text { for }\left|\frac{3}{2}, \frac{1}{2}\right\rangle .\end{cases}
\end{aligned}
$$

Here $\left\langle Y_{2}^{2}\left|Y_{1}^{1}\right| Y_{1}^{1}\right\rangle(\equiv G)$ is a Gaunt integral. In the $\left|2 p_{1 / 2}\right\rangle \rightarrow$ $\left|3 d_{x^{2}-y^{2}}\right\rangle$ and $\left|2 p_{3 / 2}\right\rangle \rightarrow\left|3 d_{x^{2}-y^{2}}\right\rangle$ XMCD absorption spectra with helicity $h=1$, the oscillator strength $\Gamma$ is finally written as follows:

$$
\begin{aligned}
& \Gamma\left(\left|\frac{1}{2}, \frac{1}{2}\right\rangle \longrightarrow Y_{2}^{2}\right)=\frac{2}{3} A G^{2}|b|^{2}\left(\equiv L_{I I}^{(1)}\right), \\
& \Gamma\left(\left|\frac{3}{2}, \frac{3}{2}\right\rangle \longrightarrow Y_{2}^{2}\right)=A G^{2}|a|^{2}\left(\equiv L_{I I I}^{(1)}\right), \\
& \Gamma\left(\left|\frac{3}{2}, \frac{1}{2}\right\rangle \longrightarrow Y_{2}^{2}\right)=\frac{1}{3} A G^{2}|b|^{2}\left(\equiv L_{I I I}^{(2)}\right),
\end{aligned}
$$

where we note that $|a|^{2}+|b|^{2}=1$. By using (23), the absorption ratio $L_{I I}^{(1)} /\left(L_{I I I}^{(1)}+L_{I I I}^{(2)}\right) \equiv \Lambda$ is calculated as

$$
\Lambda=\frac{2|b|^{2}}{3-2|b|^{2}} \text {. }
$$

The value of $|b|^{2}$ is exactly equal to $1 / 2$ when no spin current is observed. In such a situation, therefore, the value of $\Lambda$ remains $1 / 2$. However, if a sizable spin current is found, then it is sure that the value of $|b|^{2}$ differs from $1 / 2$. As can be seen from (17), the $|b|^{2}$ reflects the nature of the minority spin hole. Therefore, if the spin state of electrons injected into the superconductor is largely changed, such as the case of Figures 5(d), 5(e), and 5(f), then it could be expected that a considerable change is observed experimentally for the absorption ratio $\Lambda$ because of a large change of $|b|^{2}$. For example, let us consider the spin current at a positive voltage such as the normalized voltage 2 . In the spin current shown in Figure 5(d), the electron with the majority spin is dominant; that is, the minority spin hole is dominant, so it is expected 


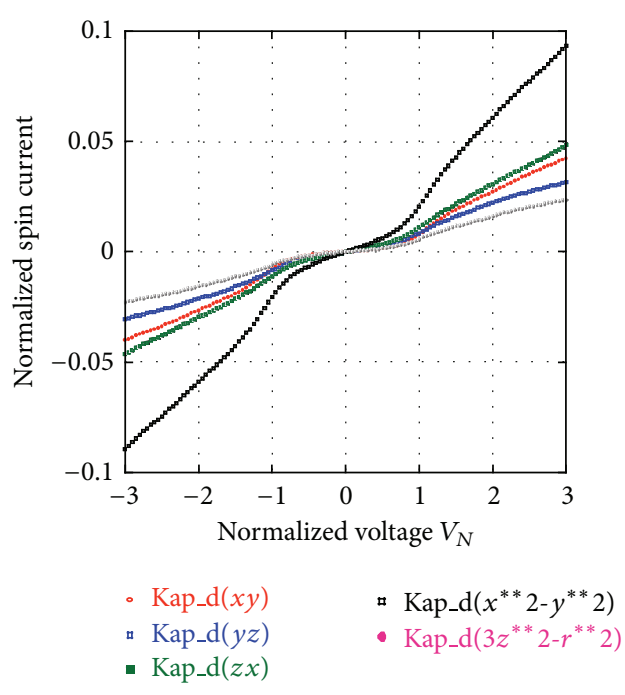

(a)

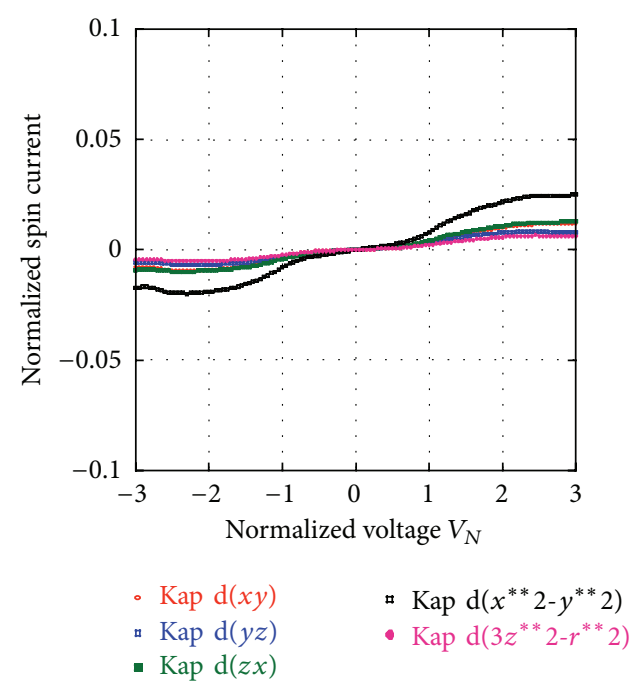

(b)

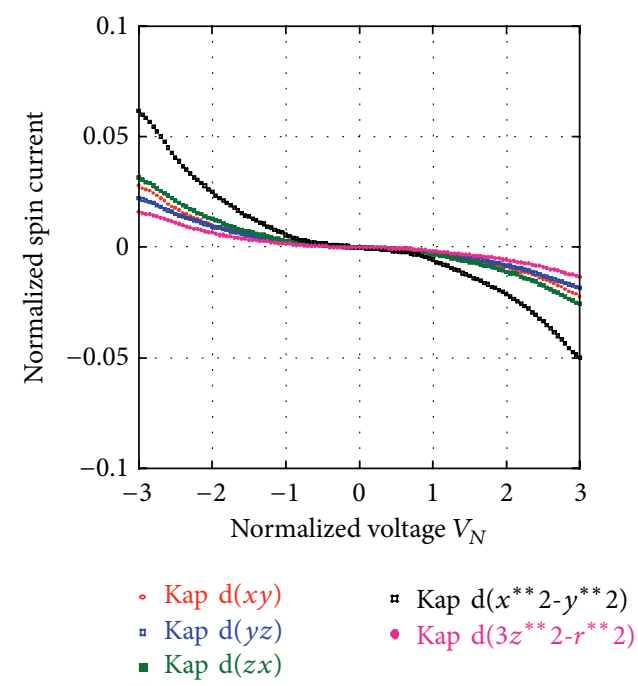

(c)

Figure 6: The $d_{x y}, d_{y z}, d_{z x}, d_{x^{2}-y^{2}}$, and $d_{3 z^{2}-r^{2}}$ atomic orbital parts of the spin currents drawn in Figures 5(d), 5(e), and 5(f). As $d$ orbitals, the $\mathrm{Cu} 3 d$ orbitals of $\mathrm{Cu} 2$ atom shown in Figure 1 have been selected. (a), (b), and (c) of the present figure correspond to Figures 5(d), 5(e), and 5(f), respectively. Present figure clearly shows that the most dominant spin current is observed in an electron tunneling between $3 d$ orbits of ferromagnetic iron and $\mathrm{Cu} 3 d_{x^{2}-y^{2}}$ orbit of $\mathrm{Hg}-1223$ high- $T_{c}$ superconductor.

that a relation $|b|^{2}>1 / 2$ is satisfied for the spin current shown in Figure 5(d). Similar consideration gives a result such that a relation $|b|^{2}<1 / 2$ is satisfied for the spin current shown in Figure 5(f). Namely, it may be expected that the absorption ratio $\Lambda$ decreases due to the change from Figures $5(\mathrm{~d})$ to $5(\mathrm{f})$, that is, with decreasing the $\eta_{\uparrow}$. It may be possible to control the value of $\eta_{\uparrow}\left(=1-\eta_{\downarrow}\right)$ by experimentally handling the amplitude of the external magnetic field applied parallel to the junction surface. It is not so easy to detect the spin current; however, we believe that present prediction based on the $\mathrm{XMCD}$ could be useful to see qualitatively the spin current found in the F/I/S tunneling junction using the copper-oxide high- $T_{c}$ superconductors.

\section{Summary}

We have studied the $c$-axis charge and spin currents in ferromagnet-insulator-superconductor (F/I/S) tunneling junction on the basis of the tunneling Hamiltonian model. As a ferromagnet, Fe metal with bcc structure has been selected for simplicity, and the electronic structure has been calculated on the basis of the spin-polarized band theory using the spin-polarized self-consistent-field atomic data for the atomic energies and the universal tight-binding parameters for the interactions. As a superconductor, $\mathrm{HgBa}_{2} \mathrm{Ca}_{2} \mathrm{Cu}_{3} \mathrm{O}_{8+\delta}(\mathrm{Hg}-$ 1223) with $\delta=0.4$ copper-oxide high- $T_{c}$ superconductor has been selected from the reason of the high $T_{c}$ of $135 \mathrm{~K}$. The 
electronic structure of $\mathrm{Hg}-1223$ has been calculated by using the same method done for the ferromagnetic Fe. The band structure calculation for Hg-1223 has clearly showed that the electronic structures near the Fermi level $E_{F}$ are made from $\mathrm{Cu} 3 d$ and $\mathrm{O} 2 p$ atomic orbitals and that the most important orbit is the $\mathrm{Cu} 3 d_{x^{2}-y^{2}}$ one in $\mathrm{CuO}_{2}$ layers in $\mathrm{Hg}-1223$.

The $c$-axis charge and spin currents in an F/I/S tunneling junction have been calculated as a function of the magnetization $\mathbf{M}_{F}$ of ferromagnet $F$ and the applied voltage $V$. Using a calculated charge current, the differential conductance has been also calculated. If the insulating layer $I$ shows a magnetic nature, it is surely expected that the tunneling probabilities of the majority and the minority spin electrons differ from each other. We have defined the $\eta_{\uparrow}$ and $\eta_{\downarrow}\left(=1-\eta_{\uparrow}\right)$ giving the tunneling probabilities of majority and the minority spin electrons. The calculated results have showed that the charge current and the resultant differential conductance nearly remain the same as the change of $\eta_{\uparrow}$, but the spin current is largely changed due to the change of $\eta_{\uparrow}$.

Present model includes the band theory, so that it is possible to see directly which atomic orbital contributes to the F/I/S tunneling phenomena. This is just an advantage of the model we have proposed here. We have clearly found that the most important orbit in the present F/I/S tunneling junction is the Cu $3 d_{x^{2}-y^{2}}$ atomic orbital in the Hg-1223 high$T_{c}$ superconductor. This is a common feature observed in not only the Hg-based superconductors but also the other copper-oxide superconductors such as YBCO and BSCCO. As a method to detect the change of the spin current, we have pointed out to the validity of the experiment of an Xray magnetic circular dichroism (XMCD).

\section{Appendix}

\section{Deduction of (1)}

First, let us consider a tunneling junction consisting of material $M 1$ /insulator $I /$ material $M 2$. For an electron tunneling from $M 1$ to $M 2$, the tunneling Hamiltonian $\widehat{H}_{T}^{1 \rightarrow 2}$ is given by

$$
\widehat{H}_{T}^{1 \rightarrow 2}=\sum_{\mathbf{k}_{1}} \sum_{\sigma_{1}} \sum_{\mathbf{k}_{2}} \sum_{\sigma_{2}}\left\langle\mathbf{k}_{2} \sigma_{2}|\widehat{T}| \mathbf{k}_{1} \sigma_{1}\right\rangle \widehat{c}_{\mathbf{k}_{2} \sigma_{2}}^{\dagger} \widehat{c}_{\mathbf{k}_{1} \sigma_{1}} .
$$

Here, $\widehat{c}_{\mathbf{k}_{1} \sigma_{1}}$ is an annihilation operator for an electron with the wavenumber $\mathbf{k}_{1}$ and spin $\sigma_{1}$ in material $M 1$ and $\widehat{c}_{\mathbf{k}_{2} \sigma_{2}}^{\dagger}$ is a creation operator for a $\mathbf{k}_{2} \sigma_{2}$-electron in $M 2 .\left\langle\mathbf{k}_{2} \sigma_{2}|\widehat{T}| \mathbf{k}_{1} \sigma_{1}\right\rangle$ is the matrix element for an electron tunneling from $\left|\mathbf{k}_{1} \sigma_{1}\right\rangle$ state to $\left|\mathbf{k}_{2} \sigma_{2}\right\rangle$ one. We consider here the case in which no spin-flip is found for an electron tunneling. Therefore, the total Hamiltonian $\widehat{H}_{T}$ defined by $\widehat{H}_{T}^{1 \rightarrow 2}+\widehat{H}_{T}^{2 \rightarrow 1}$ is given as follows:

$$
\begin{aligned}
\widehat{H}_{T}=\sum_{\mathbf{k}_{1}} \sum_{\mathbf{k}_{2}} \sum_{\sigma}\left(\left\langle\mathbf{k}_{2} \sigma|\widehat{T}| \mathbf{k}_{1} \sigma\right\rangle \widehat{c}_{\mathbf{k}_{2} \sigma}^{\dagger}{\widehat{\mathbf{k}_{1}} \sigma}_{\mathbf{k}_{1}}\right. \\
\left.+\left\langle\mathbf{k}_{1} \sigma|\widehat{T}| \mathbf{k}_{2} \sigma\right\rangle{\widehat{\mathrm{k}_{1}}}_{\mathbf{k}_{1}}^{\dagger} \widehat{\mathbf{k}}_{\mathbf{k}_{2} \sigma}\right) .
\end{aligned}
$$

Next, we consider the case such that the $M 1$ is a ferromagnetic material $F$ and the $M 2$ is a superconductor $S$, that is, an F/I/S junction. Only a superconductor in which all Cooper-pairs (CPs) are in a spin-singlet state is considered here, so it is instructive to rewrite (A.2) as follows:

$$
\begin{aligned}
& \widehat{H}_{T}=\widehat{H}_{T}^{F \rightarrow S}+\widehat{H}_{T}^{S \rightarrow F}, \\
& \widehat{H}_{T}^{F \rightarrow S}=\sum_{\mathbf{k}_{1}} \sum_{\mathbf{k}_{2}}\left(T_{\mathbf{k}_{1} \mathbf{k}_{2} \uparrow} \widehat{c}_{\mathbf{k}_{2} \uparrow}^{\dagger} \widehat{\tau}_{\mathbf{k}_{1} \uparrow}+T_{\mathbf{k}_{1} \mathbf{k}_{2} \downarrow}^{*} \widehat{c}_{-\mathbf{k}_{2} \downarrow}^{\dagger} \widehat{c}_{-\mathbf{k}_{1} \downarrow}\right) \\
& \equiv \sum_{\mathbf{k}_{1}} \sum_{\mathbf{k}_{2}} \widehat{h}_{T ; \mathbf{k}_{1} \mathbf{k}_{2}}^{F \rightarrow S}, \\
& \widehat{H}_{T}^{S \rightarrow F}=\sum_{\mathbf{k}_{1}} \sum_{\mathbf{k}_{2}}\left(T_{\mathbf{k}_{2} \mathbf{k}_{1} \uparrow} \widehat{c}_{\mathbf{k}_{1}}^{\dagger} \widehat{\uparrow}_{\mathbf{k}_{2} \uparrow}+T_{\mathbf{k}_{2} \mathbf{k}_{1} \downarrow}^{*} \widehat{\mathcal{C}}_{-\mathbf{k}_{1} \downarrow}^{\dagger} \widehat{c}_{-\mathbf{k}_{2} \downarrow}\right) \\
& \equiv \sum_{\mathbf{k}_{1}} \sum_{\mathbf{k}_{2}} \widehat{h}_{T ; \mathbf{k}_{1} \mathbf{k}_{2}}^{S \rightarrow F},
\end{aligned}
$$

where $T_{\mathbf{k}_{1} \mathbf{k}_{2} \sigma} \equiv\left\langle\mathbf{k}_{2} \sigma|\widehat{T}| \mathbf{k}_{1} \sigma\right\rangle$ and a relation $\left\langle-\mathbf{k}_{2} \sigma|\widehat{T}|-\mathbf{k}_{1} \sigma\right\rangle=$ $\left\langle\mathbf{k}_{1} \sigma|\widehat{T}| \mathbf{k}_{2} \sigma\right\rangle=\left\langle\mathbf{k}_{2} \sigma|\widehat{T}| \mathbf{k}_{1} \sigma\right\rangle^{*}$ is held. Using Bogoliubov transformation, $\widehat{h}_{T ; \mathbf{k}_{1} \mathbf{k}_{2}}^{F \rightarrow S}$ and $\widehat{h}_{T ; \mathbf{k}_{1} \mathbf{k}_{2}}^{S \rightarrow F}$ in (A.3) can be written as follows:

$$
\begin{aligned}
\hat{h}_{T ; \mathbf{k}_{1} \mathbf{k}_{2}}^{F \rightarrow S}= & T_{\mathbf{k}_{1} \mathbf{k}_{2} \uparrow}\left(u_{\mathbf{k}_{2}} \widehat{\gamma}_{\mathbf{k}_{2} \uparrow}^{\dagger} \widehat{c}_{\mathbf{k}_{1} \uparrow}+v_{\mathbf{k}_{2}}^{*} \widehat{\gamma}_{-\mathbf{k}_{2} \downarrow}{\widehat{\mathbf{k}_{1}}}_{\mathbf{k}_{1} \uparrow}\right) \\
& +T_{\mathbf{k}_{1} \mathbf{k}_{2} \downarrow}^{*}\left(u_{\mathbf{k}_{2}} \widehat{\gamma}_{-\mathbf{k}_{2} \downarrow}^{\dagger} \widehat{c}_{-\mathbf{k}_{1} \downarrow}-v_{\mathbf{k}_{2}}^{*} \widehat{\gamma}_{\mathbf{k}_{2} \uparrow} \widehat{c}_{-\mathbf{k}_{1} \downarrow}\right), \\
\widehat{h}_{T ; \mathbf{k}_{1} \mathbf{k}_{2}}^{S \rightarrow F}= & T_{\mathbf{k}_{2} \mathbf{k}_{1} \uparrow}\left(u_{\mathbf{k}_{2}}^{*} \widehat{c}_{\mathbf{k}_{1} \uparrow}^{\dagger} \widehat{\gamma}_{\mathbf{k}_{2} \uparrow}+v_{\mathbf{k}_{2}} \widehat{c}_{\mathbf{k}_{1} \uparrow}^{\dagger} \widehat{\gamma}_{-\mathbf{k}_{2} \downarrow}^{\dagger}\right) \\
& +T_{\mathbf{k}_{2} \mathbf{k}_{1} \downarrow}^{*}\left(u_{\mathbf{k}_{2}}^{*} \widehat{c}_{-\mathbf{k}_{1} \downarrow}^{\dagger} \widehat{\gamma}_{-\mathbf{k}_{2} \downarrow}-v_{\mathbf{k}_{2}} \widehat{c}_{-\mathbf{k}_{1} \downarrow}^{\dagger} \widehat{\gamma}_{\mathbf{k}_{2} \uparrow}^{\dagger}\right),
\end{aligned}
$$

where we note that $T_{\mathbf{k}_{2} \mathbf{k}_{1} \uparrow}=T_{\mathbf{k}_{1} \mathbf{k}_{2} \uparrow}^{*}$ and $T_{\mathbf{k}_{2} \mathbf{k}_{1} \downarrow}^{*}=T_{\mathbf{k}_{1} \mathbf{k}_{2} \downarrow}$. It is clear that $\widehat{\gamma}_{\mathbf{k} \sigma}^{\dagger}$ and $\widehat{\gamma}_{\mathbf{k} \sigma}$ are creation and annihilation operators for a quasiparticle characterized by $\mathbf{k} \sigma$, respectively, and that the $u_{\mathbf{k}}$ and $v_{\mathbf{k}}$ are expansion coefficients in the BCS wave function $\left|\Psi_{\mathrm{BCS}}\right\rangle=\prod_{\mathbf{k}}\left(u_{\mathbf{k}}+v_{\mathbf{k}} \widehat{c}_{\mathbf{k} \uparrow}^{\dagger} \widehat{c}_{-\mathbf{k}\rfloor}^{\dagger}\right)|0\rangle$.

Equation (A.4) tells us that an electron tunneling from $\mathbf{k}_{1}$-electron in $F$ to $\mathbf{k}_{2}$ one in $S$ can be regarded as a combination of four fundamental tunneling processes. Statistical averaging for operators such as $\widehat{\gamma}_{\mathbf{k}_{2} \uparrow}^{\dagger} \widehat{c}_{\mathbf{k}_{1} \uparrow}, \widehat{\gamma}_{-\mathbf{k}_{2} \downarrow}{\widehat{\mathbf{k}_{1}}}_{\mathbf{k}_{1}}, \widehat{\gamma}_{-\mathbf{k}_{2} \downarrow}^{\dagger} \widehat{c}_{-\mathbf{k}_{1} \downarrow} \downarrow$, and $\widehat{\gamma}_{\mathbf{k}_{2} \uparrow} \widehat{c}_{-\mathbf{k}_{1} \downarrow}$ gives the following corresponding relations:

$$
\begin{gathered}
\left\langle\hat{\gamma}_{\mathbf{k}_{2} \uparrow}^{\dagger}{\widehat{\mathbf{k}_{1} \uparrow}}_{\mathbf{k}_{1} \uparrow}\right\rangle_{\mathrm{av}}=\left(1-f\left( \pm E_{\mathbf{k}_{2} \uparrow}\right)\right) f\left(\xi_{\mathbf{k}_{1} \uparrow}^{(F)}\right), \\
\left\langle\widehat{\gamma}_{-\mathbf{k}_{2} \downarrow} \widehat{c}_{\mathbf{k}_{1} \uparrow}\right\rangle_{\mathrm{av}}=f\left( \pm E_{-\mathbf{k}_{2} \downarrow}\right) f\left(\xi_{\mathbf{k}_{1} \uparrow}^{(F)}\right), \\
\left\langle\widehat{\gamma}_{-\mathbf{k}_{2} \downarrow}^{\dagger} \widehat{c}_{-\mathbf{k}_{1} \downarrow}\right\rangle_{\mathrm{av}}=\left(1-f\left( \pm E_{-\mathbf{k}_{2} \downarrow}\right)\right) f\left(\xi_{-\mathbf{k}_{1} \downarrow}^{(F)}\right), \\
\left\langle\widehat{\gamma}_{\mathbf{k}_{2} \uparrow} \widehat{c}_{-\mathbf{k}_{1} \downarrow}\right\rangle_{\mathrm{av}}=f\left( \pm E_{\mathbf{k}_{2} \uparrow}\right) f\left(\xi_{-\mathbf{k}_{1} \downarrow}^{(F)}\right),
\end{gathered}
$$

where $\langle\cdots\rangle_{\text {av }}$ means the statistical average and $f(E)$ is FermiDirac distribution function. Here it is very instructive to point out that the symbol " \pm " in (A.6) appears when the semiconductor model is adopted for the superconductors [1]. $\xi_{\mathbf{k}_{1} \sigma}^{(F)}$ is the energy relative to the Fermi level $E_{F}^{(F)}$ of a $\mathbf{k}_{1} \sigma$ electron in ferromagnetic layer $F$, which is obtained from the band structure calculation. Here note that a relation $\xi_{-\mathbf{k}_{1} \sigma}^{(F)}=\xi_{\mathbf{k}_{1} \sigma}^{(F)}$ is satisfied for almost all the materials. $E_{\mathbf{k}_{2} \sigma}$ is 
a quasiparticle excitation energy defined by $\sqrt{\xi_{\mathbf{k}_{2} \sigma}^{(S) 2}+\left|\Delta_{\mathbf{k}_{2}}\right|^{2}}$. Here it should be noted that the superconducting energy gap $\Delta_{\mathbf{k}_{2}}$ as a function of $\mathbf{k}_{2}$ does not include explicitly the spin variable $\sigma$ since the spin-singlet CPs are considered here. $\xi_{\mathbf{k}_{2} \sigma}^{(S)}$ is the energy relative to the Fermi level $E_{F}^{(S)}$ of a $\mathbf{k}_{2} \sigma$ electron in superconducting layer $S$, which is also obtained from the band structure calculation. We consider here $\mathrm{CP}$ with a spin-singlet state, so that the $S$ should be nonmagnetic layer. Namely, the spin variable $\sigma$ of the one electron energy $\xi_{\mathbf{k}_{2} \sigma}^{(S)}$ should be dropped out; that is, a relation $\xi_{-\mathbf{k}_{2} \sigma}^{(S)}=\xi_{\mathbf{k}_{2} \sigma}^{(S)}=$ $\xi_{\mathbf{k}_{2}}^{(S)}$ should be held. A relation $\Delta_{-\mathbf{k}_{2}}=\Delta_{\mathbf{k}_{2}}$ is of course satisfied, so that a relation $E_{-\mathbf{k}_{2} \sigma}=E_{\mathbf{k}_{2} \sigma}=E_{\mathbf{k}_{2}}$ is obtained for the quasiparticle excitation energy. A similar treatment is also done for operators in (A.5).

From Fermi's golden rule, we can get the tunneling probabilities $P_{\mathbf{k}_{1} \mathbf{k}_{2}}^{F \rightarrow S}$ and $P_{\mathbf{k}_{1} \mathbf{k}_{2}}^{S \rightarrow F}$ for $F \rightarrow S$ and $S \rightarrow F$ tunneling as follows:

$$
\begin{aligned}
& P_{\mathbf{k}_{1} \mathbf{k}_{2}}^{F \rightarrow S} \\
& =\frac{2 \pi}{\hbar} \sum_{\sigma}\left|T_{\mathbf{k}_{1} \mathbf{k}_{2} \sigma}\right|^{2} \\
& \times\left\{\left(1-f\left(E_{\mathbf{k}_{2}}\right)\right) f\left(\xi_{\mathbf{k}_{1} \sigma}^{(F)}\right) \delta\left(\xi_{\mathbf{k}_{1} \sigma}^{(F)}+e V-E_{\mathbf{k}_{2}}\right)\right. \\
& \left.+f\left(E_{\mathbf{k}_{2}}\right) f\left(\xi_{\mathbf{k}_{1} \sigma}^{(F)}\right) \delta\left(\xi_{\mathbf{k}_{1} \sigma}^{(F)}+e V+E_{\mathbf{k}_{2}}\right)\right\} \\
& \equiv \sum_{\sigma} p_{\mathbf{k}_{1} \mathbf{k}_{2}, \sigma}^{F \rightarrow S}\left(\xi_{\mathbf{k}_{1} \sigma}^{(F)}, \xi_{\mathbf{k}_{2}}^{(S)}, \Delta_{\mathbf{k}_{2}}, e V\right), \\
& P_{\mathbf{k}_{1} \mathbf{k}_{2}}^{S \rightarrow F} \\
& =\frac{2 \pi}{\hbar} \sum_{\sigma}\left|T_{\mathbf{k}_{1} \mathbf{k}_{2} \sigma}\right|^{2} \\
& \times\left\{\left(1-f\left(\xi_{\mathbf{k}_{1} \sigma}^{(F)}\right)\right) f\left(E_{\mathbf{k}_{2}}\right) \delta\left(\xi_{\mathbf{k}_{1} \sigma}^{(F)}+e V-E_{\mathbf{k}_{2}}\right)\right. \\
& +\left(1-f\left(\xi_{\mathbf{k}_{1} \sigma}^{(F)}\right)\right)\left(1-f\left(E_{\mathbf{k}_{2}}\right)\right) \\
& \left.\times \delta\left(\xi_{\mathbf{k}_{1} \sigma}^{(F)}+e V+E_{\mathbf{k}_{2}}\right)\right\} \\
& \equiv \sum_{\sigma} p_{\mathbf{k}_{1} \mathbf{k}_{2}, \sigma}^{S \rightarrow F}\left(\xi_{\mathbf{k}_{1} \sigma}^{(F)}, \xi_{\mathbf{k}_{2}}^{(S)}, \Delta_{\mathbf{k}_{2}}, e V\right)
\end{aligned}
$$

Finally, we can get the charge and spin currents, $I_{T}^{(+)}(V)$ and $I_{T}^{(-)}(V)$, as a function of an applied voltage $V$. The result is given as follows:

$$
I_{T}^{( \pm)}(V) \equiv i_{T, \uparrow}(V) \pm i_{T, \downarrow}(V)
$$

and the tunneling current $i_{T, \sigma}(V)$ for the $\sigma$ spin electron is given by

$$
\begin{aligned}
& i_{T, \sigma}(V) \\
& =e \sum_{\mathbf{k}_{1}} \sum_{\mathbf{k}_{2}}\left(p_{\mathbf{k}_{1} \mathbf{k}_{2}, \sigma}^{F \rightarrow S}\left(\xi_{\mathbf{k}_{1} \sigma}^{(F)}, \xi_{\mathbf{k}_{2}}^{(S)}, \Delta_{\mathbf{k}_{2}}, e V\right)\right. \\
& \left.\quad-p_{\mathbf{k}_{1} \mathbf{k}_{2}, \sigma}^{S \rightarrow F}\left(\xi_{\mathbf{k}_{1} \sigma}^{(F)}, \xi_{\mathbf{k}_{2}}^{(S)}, \Delta_{\mathbf{k}_{2}}, e V\right)\right) \\
& =\frac{2 \pi e}{\hbar} \sum_{\mathbf{k}_{1}} \sum_{\mathbf{k}_{2}}\left|T_{\mathbf{k}_{1} \mathbf{k}_{2} \sigma}\right|^{2} \\
& \times\left\{\left(f\left(\xi_{\mathbf{k}_{1} \sigma}^{(F)}\right)-f\left(E_{\mathbf{k}_{2}}\right)\right) \delta\left(\xi_{\mathbf{k}_{1} \sigma}^{(F)}+e V-E_{\mathbf{k}_{2}}\right)\right. \\
& \quad+\left(f\left(\xi_{\mathbf{k}_{1} \sigma}^{(F)}\right)+f\left(E_{\mathbf{k}_{2}}\right)-1\right) \\
& \left.\times \delta\left(\xi_{\mathbf{k}_{1} \sigma}^{(F)}+e V+E_{\mathbf{k}_{2}}\right)\right\} \\
& \equiv \frac{2 \pi e}{\hbar} \sum_{\mathbf{k}_{1}} \sum_{\mathbf{k}_{2}}\left|T_{\mathbf{k}_{1} \mathbf{k}_{2} \sigma}\right|^{2} g\left(\xi_{\mathbf{k}_{1} \sigma}^{(F)}, \xi_{\mathbf{k}_{2}}^{(S)}, \Delta_{\mathbf{k}_{2}}, e V\right) .
\end{aligned}
$$

\section{Conflict of Interests}

The authors declare that there is no conflict of interests regarding the publication of this paper.

\section{References}

[1] G. E. Blonder, M. Tinkham, and T. M. Klapwijk, “Transition from metallic to tunneling regimes in superconducting microconstrictions: excess current, charge imbalance, and supercurrent conversion," Physical Review B, vol. 25, no. 7, pp. 4515-4532, 1982.

[2] S. Kashiwaya, Y. Tanaka, M. Koyanagi, H. Takashima, and K. Kajimura, "Origin of zero-bias conductance peaks in high-Tc superconductors," Physical Review B, vol. 51, no. 2, pp. 13501353, 1995.

[3] S. Kashiwaya, Y. Tanaka, M. Koyanagi, and K. Kajimura, "Theory for tunneling spectroscopy of anisotropic superconductors," Physical Review B, vol. 53, no. 5, pp. 2667-2676, 1996.

[4] S. Kashiwaya, Y. Tanaka, N. Yoshida, and M. R. Beasley, "Spin current in ferromagnet-insulator-superconductor junctions," Physical Review B: Condensed Matter and Materials Physics, vol. 60, no. 5, pp. 3572-3580, 1999.

[5] G. Annunziata, M. Cuoco, P. Gentile, A. Romano, and C. Noce, "Charge and spin transport through a ferromagnet/insulator/unconventional superconductor junction," Physical Review B, vol. 83, Article ID 094507, 2011.

[6] N. Takeshita, A. Yamamoto, A. Iyo, and H. Eisaki, "Zero resistivity above $150 \mathrm{~K}$ in $\mathrm{HgBa}_{2} \mathrm{Ca}_{2} \mathrm{Cu}_{3} \mathrm{O}_{8+\delta}$ at high pressure," Journal of the Physical Society of Japan, vol. 82, Article ID 023711, 2013.

[7] T. Thonhauser, H. Auer, E. Y. Sherman, and C. Ambrosch-Draxl, "First-principles study of under pressure," Physical Review B, vol. 69, Article ID 104508, 2004.

[8] C. P. Poole Jr., Handbook of Superconductivity, Academic Press, San Diego, Calif, USA, 2000. 
[9] D. J. Singh and W. E. Pickett, "Unconventional oxygen doping behavior in $\mathrm{HgBa}_{2} \mathrm{Ca}_{2} \mathrm{Cu}_{3} \mathrm{O}_{8+\delta}$," Physical Review Letters, vol. 73, p. 476, 1994.

[10] R. M. Bozorth, Ferromagnetism, IEEE Press, Piscataway, NJ, USA, 1993.

[11] A. Barone and G. Paterno, Physics and Applications of the Josephson Effects, John Wiley \& Sons, New York, NY, USA, 1982.

[12] W. A. Harrison, Elementary Electronic Structure, World Scientific, Singapore, 1999.

[13] F. Herman and S. Skillman, Atomic Structure Calculations, Prentice-Hall, Englewood Cliffs, NJ, USA, 1999.

[14] K. Schwarz, "Optimization of the statistical exchange parameter $\alpha$ for the free atoms $\mathrm{H}$ through Nb," Physical Review B, vol. 5, no. 7, pp. 2466-2468, 1972.

[15] M. Kitamura, A. Irie, and G. Oya, "Quasiparticle tunneling current-voltage characteristics of intrinsic Josephson junctions in $\mathrm{Bi}_{2} \mathrm{Sr}_{2} \mathrm{CaCu}_{2} \mathrm{O}_{8+\delta}$," Physical Review B, vol. 66, no. 5, Article ID 054519, 2002.

[16] P. M. Tedrow and R. Meservey, "Spin-dependent tunneling into ferromagnetic nickel," Physical Review Letters, vol. 26, no. 4, pp. 192-195, 1971. 

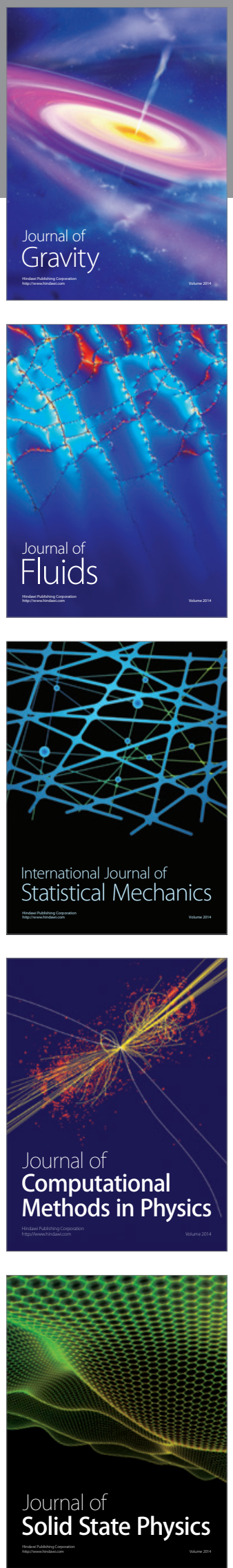

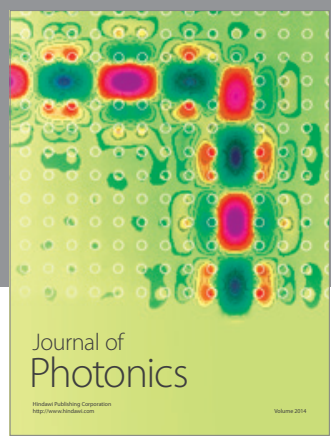

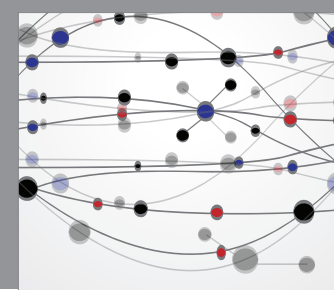

The Scientific World Journal

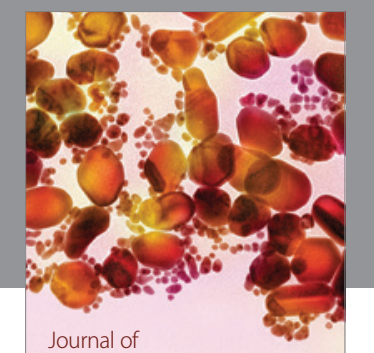

Soft Matter
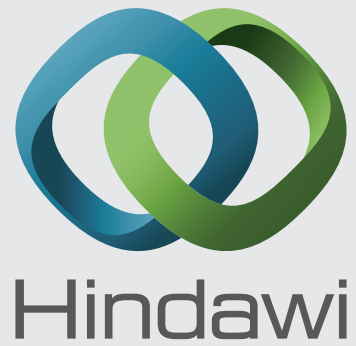

Submit your manuscripts at

http://www.hindawi.com
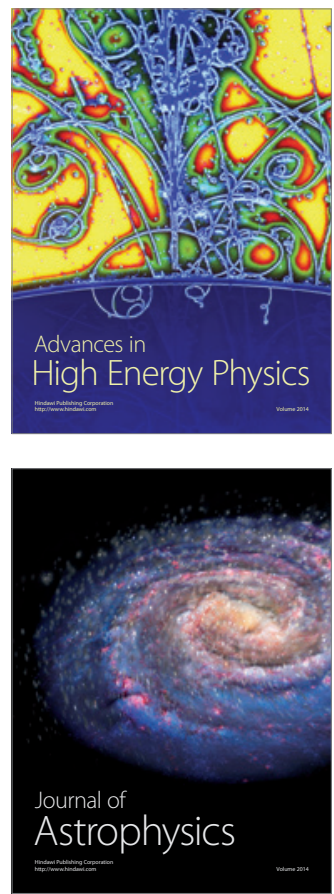
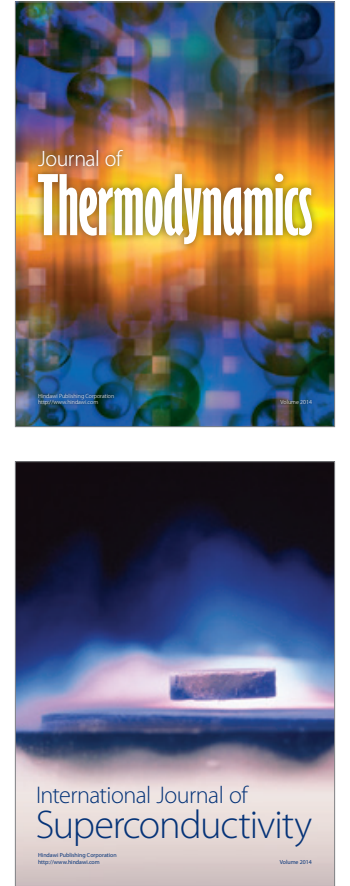
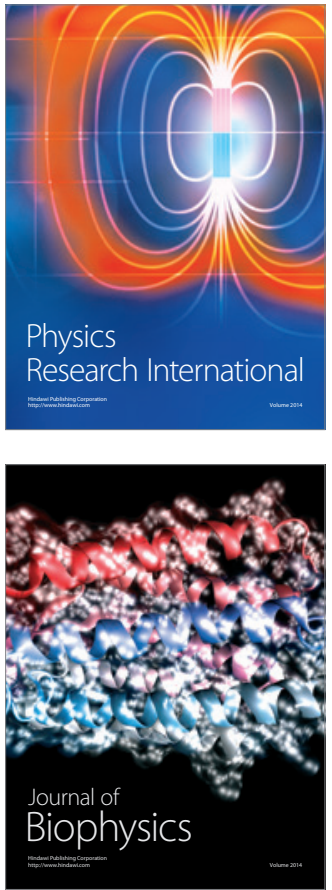
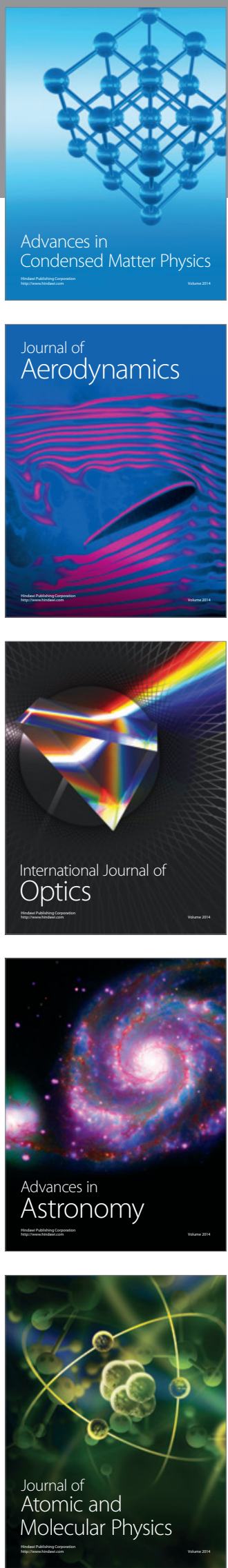